\title{
Magneto-optical absorption in silicene and germanene induced by electric and Zeeman fields
}

\author{
Do Muoi, ${ }^{1}$ Nguyen N. Hieu $\odot, 2,3,{ }^{*}$ Chuong V. Nguyen, ${ }^{4}$ Bui D. Hoi,${ }^{5}$ Hieu V. Nguyen, ${ }^{6}$ Nguyen D. Hien, \\ Nikolai A. Poklonski ${ }^{8},{ }^{8}$ S. S. Kubakaddi ${ }^{9,},{ }^{9}$ and Huynh V. Phuc ${ }^{10, \ddagger}$ \\ ${ }^{1}$ University of Science-VNU.HCM, 227 Nguyen Van Cu Street, District 5, Ho Chi Minh City 700000, Vietnam \\ ${ }^{2}$ Institute of Research and Development, Duy Tan University, Da Nang 550000, Vietnam \\ ${ }^{3}$ Faculty of Natural Sciences, Duy Tan University, Da Nang 550000, Vietnam \\ ${ }^{4}$ Department of Materials Science and Engineering, Le Quy Don Technical University, Hanoi 100000, Vietnam \\ ${ }^{5}$ Physics Department, University of Education, Hue University, Hue 530000, Vietnam \\ ${ }^{6}$ Department of Physics, The University of Da Nang, University of Science and Education, Da Nang 550000, Vietnam \\ ${ }^{7}$ Nha Trang National Ethnic Minority Pre-University, Nha Trang City, Khanh Hoa 650000, Vietnam \\ ${ }^{8}$ Physics Department, Belarusian State University, Minsk 220030, Belarus \\ ${ }^{9}$ Department of Physics, K. L. E. Technological University, Hubballi-580 031, Karnataka, India \\ ${ }^{10}$ Division of Theoretical Physics, Dong Thap University, Cao Lanh 870000, Vietnam
}

(Received 6 February 2020; revised manuscript received 31 March 2020; accepted 20 April 2020; published 7 May 2020)

\begin{abstract}
We study the optical absorption properties of silicene and germanene in the presence of the perpendicular magnetic and electric fields. Their low-energy Landau level (LL) spectra are controllable by an external electric field, where the spin- and valley-degeneracy of the LLs are strongly influenced by the electric and Zeeman fields. The electric field has removed spin-degeneracy at a given valley. Analytical expressions for the magneto-optical absorption coefficient (MOAC) are expressed in the presence of the interaction between carriers and random impurities and the intrinsic phonons including the spin and valley effects. The results evaluated for the topological insulator and valley-spin-polarized metal phases showed that when the electric field is included, the MOAC peaks are separated for opposite spin cases where the splitting in germanene is stronger than that in silicene. The peak's intensity caused by the carrier-photon-impurity scattering is the highest, the next is the carrier-photon scattering, while the carrier-photon-phonon scattering shows the lowest in both materials. Among the different phonon modes, the out-of-plane (ZA) mode in silicene dominates the others, being attributed to its buckled atomic structure, which does not exist in graphene. When the ZA mode is taken into account, the estimated resultant mobility from the full-width at half-maximum is significantly supported by the experimental result in silicene.
\end{abstract}

DOI: 10.1103/PhysRevB.101.205408

\section{INTRODUCTION}

Besides graphene [1], one of the most widely studied twodimensional (2D) materials, recently silicene and germanene, the other 2D group-IV materials, have also attracted great interest for their extraordinary properties [2,3]. Silicene and germanene have been reported not to exist in the planar geometry but in the low-buckled honeycomb structures, which have been verified by experimental observations [4,5]. Although they have low-buckled structures, silicene and germanene have the same physical properties as graphene, such as the linear band dispersion at $K$ and $K^{\prime}$ points, attributing an important feature that their charge carriers have the characteristic of massless Dirac fermions, just like graphene. Moreover, in comparison to graphene, the opening of a band gap in silicene is more convenient by applying an external electric field [6]. Besides, the spin-orbit coupling (SOC) in silicene and germanene is also bigger in comparison to that in graphene.

\footnotetext{
*Corresponding author: hieunn@duytan.edu.vn

†sskubakaddi@gmail.com

${ }^{\ddagger}$ Corresponding author: hvphuc@dthu.edu.vn
}

Therefore, the spin Hall effect in these two novel materials is stronger than that in graphene [7-9]. Another important characteristic of silicene comes from its buckled structure, which allows us to control its properties by an electric field [10], exchange field [11], or the circular polarized radiation [12]. These properties make silicene and germanene promising for wide applications, especially in the silicon-based electronics technology [13].

Spintronics and valleytronics have been playing increasingly important roles and considered as the keys of the new period of the electronics technology [9]. The spin- and valleypolarized transport have been proved to be the most vital properties of the 2D materials and have been of significant interest and studied widely [14-18]. When studying the electron transport through the barriers in monolayer $\mathrm{WSe}_{2}$, Tahir et al. [16] found that the spin splitting is essential to the valley-polarized transport. Besides, the spin-polarized current is easily controlled by the spin and valley Zeeman terms in this material. The spin-orbit interactions and spin and valley filtering have been also demonstrated to significantly affect the transport gap [14] and spin- and valley-Hall conductivities [15] in monolayer $\mathrm{MoS}_{2}$. Yuan et al. [17] studied the dependence of electron transport on the spin and valley 
polarization in monolayer $\mathrm{MoS}_{2}$. Their results showed that the spin- and valley-dependent line-type peaks occur for large incident angles, and can be adjusted by the exchange field as well as structural parameters. The orientation of circularly polarized light has been also demonstrated to be able to control the valley-dependent transmission in monolayer WSe2 [18]. Further, the spin- and valley-polarized transport properties of silicene and germanene have been of interest to study extensively [19-23]. A fully spin- and valley-polarized current has been found to oscillate with the length of the ferromagnetic silicene [19], to be controlled by an electric field [20], and to be achieved for sufficiently large electric or exchange fields [21]. When a gate voltage $U$ is taken into account, it can be used to control the spin and valley polarizations in a silicon junction system by changing its polarity [22]. By studying the intrinsic spin and valley Hall effects, Tabert and Nicol [23] showed that it is suitable to use the incident light to control the Hall conductivity in silicene and germanene.

The magneto-optical absorption is one of the important properties studied in the literature in bulk semiconductors [24], low-dimensional systems [25-27], and more recently in monolayers of graphene [28] and transition-metal dichalcogenides (TMDCs) [29-31]. In this phenomenon, in quantizing magnetic field, optical absorption takes place by absorbing a photon and making inter-Landau level transition with/without the assistance of impurity or phonons scattering. The energy levels of the TMDCs are governed by the product of the valley index, $\tau$, and the spin index, $s$, i.e., depending on the product $s \tau$. This means that the bands of $K$ and $K^{\prime}$ valleys can be corresponded by interchanging the spin directions. Therefore, the four transitions in monolayer TMDCs could be divided into two pairs, $K \uparrow\left(K^{\prime} \downarrow\right)$ and $K \downarrow\left(K^{\prime} \uparrow\right)$ because of their spin-valley coupling. Although rigorous study of the valley and spin-polarized transport [19-23] and electrical transport [32-36] properties are underway in silicene and in germanene [37], the MOAC has been not addressed in these materials yet. It is expected that the MOAC in these materials to be fairly different from that in TMDCs due to different band structure.

In this paper, we study the magneto-optical absorption in silicene and germanene in quantizing magnetic field. We derive and discuss their Landau levels (LLs) structure in the presence and absence of electric and Zeeman fields. The magneto-optical absorption coefficients (MOACs) are evaluated for three values of electric field including the interaction between the carriers and impurities $[38,39]$ and phonons [40-43]. The impurity scattering is considered in the short-ranged potential case [44] and is demonstrated to play a substantial role, especially in the low-temperature regime. As for the phonon scattering, all three acoustic [outof-plane (ZA), transverse (TA), and longitudinal (LA)] and optical (ZO, TO, LO) phonon modes of silicene $[32,42,45]$ are included and discussed meticulously, making the present work's results much more fulfilled than our previous works [29,31]. The full-width at half-maximum (FWHM) of the resonant peaks is obtained by the computational method and discussed in detail. When the ZA mode is taken into account, the predicted resultant mobility in silicene, which is obtained from the FWHM data, has been found to reduce from 1218 to $285.8 \mathrm{~cm}^{2} /(\mathrm{V} \mathrm{s})$, which is in agreement with experimental observation at $T=300 \mathrm{~K}$ in silicene.
The paper is organized as follows. In Sec. II we present the basic information including the eigenfunctions and eigenvalues and the interaction mechanisms. In Sec. III we present the analytical results for the MOAC expressions in both two- and three-particle scatterings. The numerical results are presented in Sec. IV. Finally, our conclusions and summary are presented in Sec. V.

\section{BASIC FORMULATION}

We consider a silicene/germanene sheet oriented in the (xy) plane with a magnetic field $\mathbf{B}=(0,0, B)$ applied to the system. Including the Zeeman field, $M_{z}=g \mu_{B} B / 2$ with $g=4$ and $\mu_{B}=e \hbar / 2 m_{0}$ being the electron spin factor and Bohr magneton [46], respectively, to the effective low-energy Hamiltonian for carriers of Ref. [39], we have

$$
\mathcal{H}_{e}=v_{F}\left(\tau \sigma_{x} \pi_{x}+\sigma_{y} \pi_{y}\right)-\left(\tau s \lambda_{\text {SO }}-\Delta_{z}\right) \sigma_{z}+s M_{z} .
$$

Here, $v_{F}$ presents the Fermi velocity, $\tau= \pm 1$ is the valley index, the terms $\sigma_{x}, \sigma_{y}, \sigma_{z}$ are the Pauli matrices, $\lambda_{\text {so }}$ is the strength of SOC, $\Delta_{z}=e d E_{z}$, with $E_{z}$ being the perpendicular applied electric field, and $2 d$ being the vertical distance between the two sublattices. For visual understanding of silicene structure, the readers are referred to Fig. 1 of Ref. [39] for more detail. Furthermore, $\pi=\mathbf{p}+e \mathbf{A}$ is the momentum operator with the vector potential $\mathbf{A}=(0, B x, 0), s= \pm 1$ is the spin index. It is noted that there is also a Rashba SOC interaction $[9,11,47]$, however, it is typically neglected because of its small magnitude. Note that the effective Hamiltonian Eq. (1) has the same form of that in TMDCs systems [31] because both TMDCs and silicene as well as germanene are the $2 \mathrm{D}$ systems. The main differences between them come from the energy gap and the SOC terms. While in TMDCs the large natural band gap is the cause of their large energy gap term (for example, $\Delta=0.83 \mathrm{eV}$ in $\mathrm{MoS}_{2}$, see Table I of Ref. [31]), there is a complete lack of this term, i.e., $\Delta=0$, in silicene and germanene as shown in Eq. (1).

The wave functions corresponding to Eq. (1) can be expressed in the form $\psi\left(\mathbf{r}, k_{y}\right)=e^{i k_{y} y} \psi(x) / \sqrt{L_{y}}$ while the eigenvalues are

$$
E_{\xi}=E_{n, s}^{\tau, p}=p\left[n\left(\hbar \omega_{c}\right)^{2}+\left(\Delta_{\tau, s}^{z}\right)^{2}\right]^{1 / 2}+s M_{z} .
$$

Here the electronic state is presented through the quantum numbers as $|\xi\rangle=\left|n, s, \tau, p, k_{y}\right\rangle, p= \pm 1$ is the band index for conduction and valence bands, $n(n \geqslant 1)$ is the Landau level index, $\Delta_{\tau, s}^{z}=-\tau s \lambda_{\text {SO }}+\Delta_{z}$, and $\omega_{c}=v_{F} \sqrt{2} / \alpha_{c}$ is the cyclotron frequency with the magnetic length $\alpha_{c}=(\hbar / e B)^{1 / 2}$ The associated eigenfunctions are

$$
\begin{aligned}
& \psi_{n, s}^{+1, p}(x)=\left(\begin{array}{c}
C_{n, s}^{+1, p} \phi_{n-1}\left(x-x_{0}\right) \\
D_{n, s}^{+1, p} \phi_{n}\left(x-x_{0}\right)
\end{array}\right), \\
& \psi_{n, s}^{-1, p}(x)=\left(\begin{array}{c}
-C_{n, s}^{-1, p} \phi_{n}\left(x-x_{0}\right) \\
D_{n, s}^{-1, p} \phi_{n-1}\left(x-x_{0}\right)
\end{array}\right),
\end{aligned}
$$

where $C_{n, s}^{\tau, p}$ and $D_{n, s}^{\tau, p}$ are the normalization constants. Furthermore, $\phi_{n}\left(x-x_{0}\right)$ are the harmonic oscillator wave functions centered at $x_{0}=\alpha_{c}^{2} k_{y}$. The eigenvalues for $n=0$ are

$$
E_{0, s}^{\tau}=-\tau s \lambda_{\mathrm{SO}}+\Delta_{z}+s M_{z} .
$$



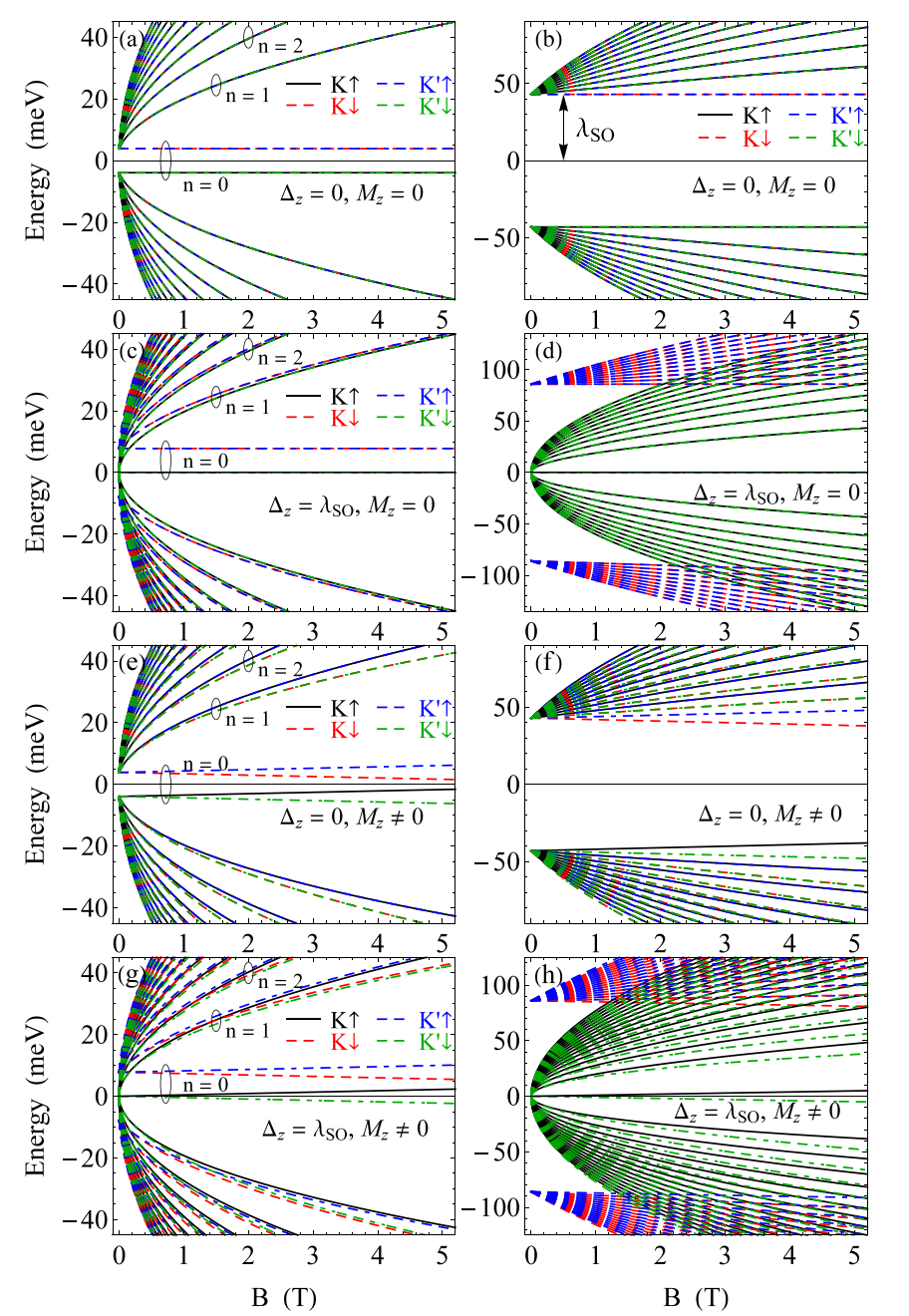

FIG. 1. The LLs of silicene [the left panels: (a), (c), (e), (g)] and germanene [the right panels: (b), (d), (f), (h)] versus the magnetic field.

Unlike the case of $n \neq 0$, where each value of LL index, $n$, includes two values of $p$ corresponding to electrons $(p>0)$ and holes $(p<0)$, the lowest OLL is for both electrons and holes with the shared eigenfunctions $\psi_{0, s}^{+1}=\left(0, \phi_{0}\right)^{T r}$ and $\psi_{0, s}^{-1}=\left(\phi_{0}, 0\right)^{T r}$, where $\operatorname{Tr}$ refers to the transpose operator.

Depending on the value of the ratio $\Delta_{z} / \lambda_{\text {so }}$ the system is referred to three different phases: if $\Delta_{z} / \lambda_{\text {so }}<1$ the system shows topological insulator (TI) phase, $\Delta_{z} / \lambda_{\mathrm{SO}}=1$ is for the valley-spin-polarized metal (VSPM) phase, and $\Delta_{z} / \lambda_{\text {SO }}>1$ the system is referred to as the band insulator (BI) phase, respectively [46]. In Fig. 1, we show the magnetic fielddependent LLs of silicene and germanene in both with (VSPM phase) and without (TI phase) electric and Zeeman fields using parameters in Table I. For (i) $\Delta_{z}=M_{z}=0$ [see Figs. 1(a) and 1(b)], all LLs with $n \neq 0$ in both silicene and germanene are quadruple spin and valley degenerate, while the lowest LLs $n=0$ are twice degenerate and are independent of magnetic field. The LLs for the conduction $(p=+1)$ and valence $(p=-1)$ bands are symmetrical through the centerline $(E=$ $0)$. With the stronger SOC, the spin splitting in germanene is larger in comparison to that in silicene. For (ii) $\Delta_{z}=\lambda_{\text {SO }}$
TABLE I. List of material parameters. Values of $a(\AA), v_{F}\left(\times 10^{5}\right.$ $\mathrm{m} / \mathrm{s})$, and $\lambda_{\text {So }}(\mathrm{meV})$ are taken from Ref. [48], $d(\AA)$ is taken from Ref. [11], $m_{e}$ (in units of $m_{0}$ ) is taken from Refs. [49,50], and the relative dielectric constant $\varepsilon$ is taken from Refs. [39,51].

\begin{tabular}{lcccrcc}
\hline \hline & $a$ & $v_{F}$ & $d$ & $\lambda_{\text {so }}$ & $m_{e}$ & $\varepsilon$ \\
\hline Silicene & 3.86 & 5.42 & 0.23 & 3.9 & 0.26 & 4.0 \\
Germanene & 4.02 & 5.24 & 0.33 & 43.0 & 0.12 & 2.4 \\
\hline \hline
\end{tabular}

and $M_{z}=0$ [see Figs. $1(\mathrm{c})$ and $1(\mathrm{~d})$ ], each LL is twice spin degenerate with $K \uparrow=K^{\prime} \downarrow$ and $K \downarrow=K^{\prime} \uparrow$. Furthermore, the LLs spectrum for the spin-up of $K$ valley $(K \uparrow)$ and the spindown of $K^{\prime}$ valley $\left(K^{\prime} \downarrow\right)$ in both silicene and germanene are like those of the pristine graphene monolayer. This is because the value of electric field $E_{z}=\lambda_{\mathrm{SO}} / e d$ canceled the SOC terms for $K \uparrow$ and $K^{\prime} \downarrow$. The results will be for $K \downarrow$ and $K^{\prime} \uparrow$ if the direction of $E_{z}$ is reversed, being in agreement with those reported in unmodulated silicene [39]. For (iii) $\Delta_{z}=0$ and $M_{z} \neq 0$ shown in Figs. 1(e) and 1(f), the LLs (except for $n=$ $0)$ are twice valley degenerate, i.e., $K \uparrow=K^{\prime} \uparrow$ and $K \downarrow=K^{\prime} \downarrow$. Due to the Zeeman field effect, each of the LLs, in this case, is separated into two levels for spin-up and spin-down. For (iv) the case of $\Delta_{z}=\lambda_{\text {so }}$ and $M_{z} \neq 0$ shown in Figs. $1(\mathrm{~g}$ ) and $1(\mathrm{~h})$, the results obtained here are the combination of the results of the second and the third cases: the electric field cancels the SOC terms and the Zeeman term separates the LLs due to spin-up and spin-down. In this case, the LLs are separated and are nondegenerate. This means that the electric field has broken the spin degeneracy at the given valley. Note that, at this critical electric field value $\left(\Delta_{z}=\lambda_{\mathrm{SO}}\right)$, the 0LL at each valley rolls into the Dirac point, which refers to a new type of metal phase called the VSPM phase $[11,46]$.

Now, with the propagation of an electromagnetic field (or radiation) polarized in the plane of the layer and assuming carriers to interact with the random impurities and phonons, the total Hamiltonian is

$$
\mathcal{H}=\mathcal{H}_{e}+\mathcal{H}_{p h}+\mathcal{H}_{e p t}+\mathcal{H}_{e i}+\mathcal{H}_{e p},
$$

where $\mathcal{H}_{e p t}=\left(-e / m_{e}\right)(\pi \cdot \mathbf{A})$ is the electron-photon interaction energy. The phononic part of Hamiltonian in Eq. (6) is

$$
\mathcal{H}_{p h}=\sum_{\mathbf{q}, v} \hbar \omega_{\mathbf{q}, v} b_{\mathbf{q}, v}^{\dagger} b_{\mathbf{q}, v}
$$

where $b_{\mathbf{q}, v}^{\dagger}$ and $b_{\mathbf{q}, v}$ are the creation and annihilation operators of phonon, respectively, and $\hbar \omega_{\mathbf{q}, v}$ is the phonon energy. The interaction parts of Hamiltonian (6), between carriers with impurities and phonons, are given as follows [52]:

$$
\begin{gathered}
\mathcal{H}_{e i}=\sum_{\mathbf{q}, a} \sum_{\xi, \xi^{\prime}} U(q) J_{\xi, \xi^{\prime}}(q) e^{i \mathbf{q}\left(\mathbf{r}-\mathbf{r}_{a}\right)} c_{\xi}^{\dagger} c_{\xi^{\prime}}, \\
\mathcal{H}_{e p}=\sum_{\mathbf{q}, v} \sum_{\xi, \xi^{\prime}} \mathrm{g}_{\mathbf{q}}^{\nu} J_{\xi, \xi^{\prime}}(q) e^{i \mathbf{q r}} c_{\xi}^{\dagger} c_{\xi^{\prime}}\left(b_{-\mathbf{q}, v}^{\dagger}+b_{\mathbf{q}, v}\right) .
\end{gathered}
$$

Here, $U(q)$ and $\mathbf{g}_{\mathbf{q}}^{v}$ are the scattering potentials between carriers and impurity and the carrier-phonon coupling matrix, respectively. The term $\mathbf{r}_{a}$ is the position of impurity. The notation $c_{\xi}^{\dagger}\left(c_{\xi^{\prime}}\right)$ is the carrier creation (annihilation) operator, 
and the form factor is

$$
J_{\xi, \xi^{\prime}}(q)=\int_{-\infty}^{+\infty} e^{i q_{x} x} d x\left[\psi_{n^{\prime}, s^{\prime}}^{\tau^{\prime}, p^{\prime}}(x)\right]^{\dagger} \psi_{n, s}^{\tau, p}(x),
$$

which has the same form as Eq. (A1) of Ref. [31], but with the eigenfunctions shown in Eqs. (3) and (4).

\section{MAGNETO-OPTICAL RESPONSE}

This section is devoted to introduce the general formalism to evaluate the magneto-optical absorption coefficients in both cases: with and without the scattering between carriers and impurities and/or phonons. Here, we only study the undoped silicene and germanene, where the Fermi level locates at the zero level. This means that only optical interband transitions from the valence $(v)$ to the conduction bands $(c)$ can occur while the intraband transitions are forbidden.

\section{A. Two-particle scattering case}

When the scattering between carriers and impurities and/or phonons is not taken into account we have the twoparticle scattering case, i.e., we only study the interaction between the carrier and photon. In this case, the MOAC can be given as follows [53]:

$$
K^{0}(\Omega)=\frac{2 \pi \Omega}{c \varepsilon_{0} V_{0}} \sum_{c, v} \sum_{k_{y}, k_{y}^{\prime}}\left|\mathcal{M}_{c v}^{0}\right|^{2} \delta\left(E_{c}-E_{v}-\hbar \Omega\right),
$$

where we have used MKS units instead of the CGS-units as done in the Ref. [53]. In Eq. (11), $\varepsilon_{0}$ is the vacuum permittivity, $\Omega$ is the incident photon frequency, $c$ is the light speed, $V_{0}$ is the sample volume, $E_{c}\left(E_{v}\right)$ is the energy shown in Eq. (2) for the conduction (valence) band. The optical transition matrix element is

$$
\mathcal{M}_{c v}^{0}=\frac{e \hbar}{m_{e}} \frac{\left\langle c ; k_{y}^{\prime}\left|p_{x}\right| v ; k_{y}\right\rangle}{E_{c}-E_{v}}=\frac{e \hbar}{m_{e}} \frac{\mathcal{B}_{c, v}}{E_{c, v}} \delta_{k_{y}^{\prime}, k_{y}},
$$

with $e$ being the electron charge, $\mathcal{B}_{c, v}=\left\langle c\left|p_{x}\right| v\right\rangle$ is the dipole moment with $p_{x}=-i \hbar \partial / \partial x$ being the carrier momentum operator in the $x$ direction, and $E_{c, v}=E_{c}-E_{v}$ denotes the LLs separation between conduction and valence bands.

To evaluate the sum over $k_{y}$, we use the following prescription $\sum_{k_{y}} \rightarrow\left(L_{y} / 2 \pi\right) \int_{-L_{z} / 2 \alpha_{c}^{2}}^{L_{x} / 2 \alpha_{c}^{2}} d k_{y}=\mathcal{A} / 2 \pi \alpha_{c}^{2}$ with $\mathcal{A}=L_{x} L_{y}$ being the sample area. Inserting Eq. (12) into Eq. (11) we have

$$
K^{0}(\Omega)=\frac{2 \pi \hbar \alpha_{S}}{m_{e}^{2} \Omega d \alpha_{c}^{2}} \sum_{c, v}\left|\left\langle c\left|p_{x}\right| v\right\rangle\right|^{2} \delta\left(E_{c, v}-\hbar \Omega\right),
$$

where $\alpha_{S}=e^{2} / 4 \pi \varepsilon_{0} \hbar c$ is the Sommerfeld's fine structure constant. Using Eqs. (3) and (4), the expression for the dipole moment, $\mathcal{B}_{c, v}=\left\langle c\left|p_{x}\right| v\right\rangle$, is expressed as follows:

$$
\begin{aligned}
\mathcal{B}_{c, v}= & \frac{i \hbar}{\alpha_{c} \sqrt{2}}\left(C_{n^{\prime}, s^{\prime}}^{\tau^{\prime},+1} C_{n, s}^{\tau,-1}+D_{n^{\prime}, s^{\prime}}^{\tau^{\prime},+1} D_{n, s}^{\tau,-1}\right) \\
& \times\left(\sqrt{n+1} \delta_{n^{\prime}, n+1}-\sqrt{n} \delta_{n^{\prime}, n-1}\right) \delta_{s, s^{\prime}} \delta_{k_{y}, k_{y}^{\prime}},
\end{aligned}
$$

where the relation $|\tau|^{2}=\left|\tau^{\prime}\right|^{2}=1$ is used. Finally, using the Lorentzian representation of the delta function, i.e., $\delta(x) \rightarrow$ $(\gamma / \pi)\left(\gamma^{2}+x^{2}\right)$ with $\gamma \propto \sqrt{B}(\mathrm{meV})$, the absorption coefficient in Eq. (13) can be evaluated.

\section{B. Three-particle scattering case}

When the interaction between carriers and impurities and/or phonons is taken into account we have the case of the interaction of three particles (carrier, photon, and impurity/phonon), and the MOAC can be evaluated as follows [54-56]:

$$
K^{\eta}(\Omega)=\frac{\sqrt{\varepsilon}}{N_{\Omega} c} \sum_{c, v} \sum_{k_{y}, k_{y}^{\prime}} \mathcal{W}_{c, v}^{\eta, \pm} f_{v}\left(1-f_{c}\right) .
$$

Here, the index $\eta$ refers to im or $v$ for impurity and/or phonon scatterings, respectively. The term $\varepsilon$ is the dielectric constant, $N_{\Omega}$ is the number of incident photons of energy $\hbar \Omega$, and $f_{v(c)}$ is the carrier distribution function at the valence (conduction) band. The term $\left(1-f_{c}\right)$ implies that the final states at the conduction band are unoccupied. The transition matrix element is $[57,58]$

$$
\begin{aligned}
\mathcal{W}_{c, v}^{\eta, \pm}= & \frac{2 \pi}{\hbar^{3} \Omega^{2}} \sum_{i, k_{y}^{\prime \prime}} \sum_{\mathbf{q}} \sum_{k=1}^{\infty}\left|\mathcal{M}_{c, i}^{\eta, \pm}\right|^{2}\left|\mathcal{M}_{i, v}^{p t}\right|^{2} \\
& \times \frac{\left(\alpha_{0} q\right)^{2 k}}{(k !)^{2} 2^{2 k}} \delta\left(E_{c}-E_{v} \pm \hbar \omega_{\mathbf{q}}^{\eta}-k \hbar \Omega\right),
\end{aligned}
$$

where the $+(-)$ sign corresponds to the phonon emission (absorption) process. The quantity $\hbar \omega_{\mathbf{q}}^{\eta}$ is equal to zero for impurity and/or $\hbar \omega_{\mathbf{q}}^{v}$ for phonon scattering, respectively, $k$ is the number of absorbed photons, and $\alpha_{0}=7 \mathrm{~nm}$ refers to the dressing parameter [29]. For the physical meaning of the transition matrix element, the readers are referred to Ref. [31] for further details, where we have redenoted the initial, intermediate, and final states as $|\alpha\rangle \equiv\left|v ; k_{y}\right\rangle,\left|\alpha^{\prime \prime}\right\rangle \equiv$ $\left|i ; k_{y}^{\prime \prime}\right\rangle$, and $\left|\alpha^{\prime}\right\rangle \equiv\left|c ; k_{y}^{\prime}\right\rangle$, respectively. In Eq. (16), $\mathcal{M}_{c, i}^{\eta, \pm}$ and $\mathcal{M}_{i, v}^{p t}$ describe the two separation parts of the interactions: carrier-impurity/phonon and carrier-photon, respectively. The former is

$$
\left|\mathcal{M}_{c, i}^{\eta, \pm}\right|^{2}=N_{\mathbf{q}}^{\eta, \pm}\left|\mathrm{g}_{\mathbf{q}}^{\eta}\right|^{2} \cos ^{2}\left(\frac{\theta}{2}\right)\left|J_{c, i}(q)\right|^{2} \delta_{s^{\prime}, s^{\prime \prime}} \delta_{k_{y}^{\prime \prime}}, k_{y}^{\prime} \mp q_{y} .
$$

Here, the quantity $N_{\mathbf{q}}^{\eta, \pm}$ is $N_{i}$ for the impurity scattering, with $N_{i}=1 \times 10^{9} \mathrm{~cm}^{-2}$ being the impurity density [39], and is $N_{\mathbf{q}}^{v, \pm}$ for the phonon scattering, with $N_{\mathbf{q}}^{v, \pm}=N_{\mathbf{q}}^{v}+1 / 2 \pm 1 / 2$ ( $N_{\mathbf{q}}^{v}$ is the phonon distribution function), and $\theta$ denotes the scattering angle. Furthermore, the term $\mathrm{g}_{\mathbf{q}}^{\eta}$ stands for $U(q)$ for impurity and/or $\mathrm{g}_{\mathbf{q}}^{v}$ for phonon interactions, respectively. The form factor $J_{c, i}(q)$ is shown in Eq. (10), where the symbol $c(i)$ stands for the final (intermediate) state $\xi^{\prime}\left(\xi^{\prime \prime}\right)$.

In Eq. (16), the carrier-photon interaction part is given by $[55,59]$

$$
\mathcal{M}_{i, v}^{p t}=-\frac{e \hbar}{m_{e}}\left(\frac{2 \pi N_{\Omega}}{\varepsilon V_{0} \hbar \Omega}\right)^{1 / 2} \mathcal{B}_{i, v},
$$

where $\mathcal{B}_{i, v}=\left\langle i\left|p_{x}\right| v\right\rangle$ is the dipole moment for the valence and intermediate bands.

\section{Carrier-photon-impurity scattering}

For the carrier-photon-impurity scattering, we have $\hbar \omega_{\mathbf{q}}^{\eta}=$ 0 and $\mathrm{g}_{\mathbf{q}}^{\eta}=U(q)$, where the intravalley scattering potential is 
$[38,39]$

$$
U(q)=\frac{U_{0}}{\left(q^{2}+q_{0}^{2}\right)^{1 / 2}} .
$$

Here $U_{0}=e^{2} /\left(2 \mathcal{A} \varepsilon_{0} \varepsilon\right)$ and $q_{0}=e^{2} \sqrt{n_{e}} / \sqrt{\pi} \varepsilon_{0} \varepsilon \hbar v_{F}$ is the screening wave vector [44]. For the carrier density of $n_{e}=$ $3 \times 10^{12} \mathrm{~cm}^{-2}$ [43] we attain $q_{0}=1.24 \times 10^{9} \mathrm{~m}^{-1}$ in silicene and $q_{0}=2.13 \times 10^{9} \mathrm{~m}^{-1}$ in germanene, respectively. Therefore, in the short-ranged potential case, it is suitable to use the following reduced expression $U(q) \approx U_{0} / q_{0}$, which is the result of the relation $q \ll q_{0}$. Using these formulas in Eq. (15), the MOAC expression for the carrier-photonimpurity scattering is found

$$
\begin{aligned}
K^{i m}(\Omega)= & \frac{4 N_{i} U_{0}^{2}}{q_{0}^{2} \alpha_{c}^{4}} \sum_{c, v, i} F_{c, v, i}\left\{\mathcal{G}_{c, i}^{(1)} \delta\left(E_{c, v}-\hbar \Omega\right)\right. \\
& \left.+\frac{\alpha_{0}^{2}}{8 \alpha_{c}^{2}} \mathcal{G}_{c, i}^{(2)} \delta\left(E_{c, v}-2 \hbar \Omega\right)\right\},
\end{aligned}
$$

where the sum over $k$ in Eq. (16) is taken from 1 to 2 for the one- and two-photon absorption processes, and

$$
F_{c, v, i}=\frac{\mathcal{A} e^{2} \alpha_{0}^{2}}{16 c m_{e}^{2} \sqrt{\varepsilon} \hbar^{2} \Omega^{3} d \alpha_{c}^{2}}\left|\mathcal{B}_{i, v}\right|^{2} f_{v}\left(1-f_{c}\right) .
$$

Here, the dipole moment $\mathcal{B}_{i, v}$ is calculated as the same form as Eq. (14) but with $c$ state replaced by $i$ state. The dimensionless integral $\mathcal{G}_{c, i}^{(k)}$ in Eq. (20) is defined as

$$
\mathcal{G}_{c, i}^{(k)}=\int_{0}^{\infty} u^{k}\left|J_{c, i}(u)\right|^{2} \mathrm{~d} u, u=\alpha_{c}^{2} q^{2} / 2,
$$

which would be analytically evaluated by using Eqs. (A1)(A4) of Ref. [60]. The Dirac functions in Eq. (20) are approximated by the Lorentzians of width $\Gamma_{c, v}^{i m}$, where $\left(\Gamma_{c, v}^{i m}\right)^{2}=$ $\left(\mathcal{A} U_{0}^{2} / 2 \pi q_{0}^{2} \alpha_{c}^{2}\right) \mathcal{G}_{c, v}^{(0)} \delta_{s, s^{\prime}}$ is obtained using the collisionbroadening model [61]. Here, $\mathcal{G}_{c, v}^{(0)}$ is given in Eq. (22) for $k=0$ and the $i$ state is replaced by the $v$ state. This clearly indicates that the Lorentz width, $\Gamma_{c, v}^{i m}$, is proportional to $\left(B / n_{e}\right)^{1 / 2}$, which looks identical to that of the LL broadening in graphene [62].

\section{Carrier-photon-phonon scattering}

For the carrier-photon-phonon scattering, we have $\hbar \omega_{\mathbf{q}}^{\eta}=$ $\hbar \omega_{\mathbf{q}}^{\nu}$ and $\mathrm{g}_{\mathbf{q}}^{\eta}=\mathrm{g}_{\mathbf{q}}^{v}$. The deformation potential theory of electron-phonon coupling in buckled silicene and germanene is discussed in Refs. [32,36,41]. According to that, the coupling matrix element, for the deformation potential, is given by

$$
\left|\mathrm{g}_{\mathbf{q}}^{\nu}\right|^{2}=\frac{\hbar}{2 \mathcal{A} \rho \omega_{\mathbf{q}, v}^{\lambda}}\left|D_{\mathbf{q}, v}^{\lambda}\right|^{2}
$$

where $\rho=7.2 \times 10^{-7}\left(1.6 \times 10^{-6}\right) \mathrm{kg} / \mathrm{m}^{2}$ is the mass density of silicene (germanene) [42]. Furthermore, the electronphonon coupling strength can be expressed in the first order $D_{\mathbf{q}, \text { ac }}^{\lambda}=D_{1}^{\lambda} q$ for the acoustic phonon $(v=\mathrm{ac})$ or in the zeroth order $D_{\mathbf{q}, \text { op }}^{\lambda}=D_{0}^{\lambda}$ for the optical phonon $(v=$ op), respectively. Here, the phonon mode indices $\lambda$ refer to ZA, TA, LA, or ZO, TO, and LO.
For the acoustic phonon interaction, the LA and TA branches of silicene and germanene are linear while the outof-plane (ZA) branch is nonlinear in the vicinity of $\Gamma$ point [40,42]. However, in a previous work, Kuang et al. observed that even the ZA branch of group-IV buckled monolayers is also the linear dispersion [63]. Therefore, in this work, we assume a simple linear frequency dispersion model, $\omega_{\mathbf{q}, a c}^{\lambda}=$ $v_{s}^{\lambda} q$, for all three acoustic modes. Here, $v_{s}^{\lambda}$ is the sound velocity. Then, the MOAC in Eq. (15) will turn out to be

$$
\begin{aligned}
K^{\mathrm{ac}}(\Omega)= & \frac{2 k_{B} T D_{1}^{2}}{V_{0} \rho v_{s}^{2} \alpha_{c}^{4}} \sum_{c, v, i} F_{c, v, i}\left\{\mathcal{G}_{c, i}^{(1)} \delta\left(E_{c, v}-\hbar \Omega\right)\right. \\
& \left.+\frac{\alpha_{0}^{2}}{8 \alpha_{c}^{2}} \mathcal{G}_{c, i}^{(2)} \delta\left(E_{c, v}-2 \hbar \Omega\right)\right\},
\end{aligned}
$$

where the phonon energy in the arguments of Dirac functions is neglected due to its much smaller values in comparison to $E_{c, v}, k_{B} T$ is the thermal energy, the dimensionless integrals $\mathcal{G}_{c, i}^{(1,2)}$ are defined in Eq. (22) for $k=1,2$. The corresponding Lorentz width is $\left(\Gamma_{c, v}^{\mathrm{ac}}\right)^{2}=\left(D_{1}^{2} k_{B} T / 4 \pi \rho v_{s}^{2} \alpha_{c}^{2}\right) \mathcal{G}_{c, v}^{(0)} \delta_{s, s^{\prime}}$.

The MOAC expression for the optical phonon interaction is found as follows:

$$
K^{\mathrm{op}}(\Omega)=\frac{\hbar D_{0}^{2}}{V_{0} \rho \omega_{\mathrm{op}} \alpha_{c}^{4}} \sum_{c, v, i} F_{c, v, i}\left(\mathcal{P}_{1}+\mathcal{P}_{2}\right),
$$

where we have used the dispersionless frequency, $\omega_{\mathbf{q}}^{\mathrm{op}}=\omega_{\mathrm{op}}$, and

$$
\begin{gathered}
\mathcal{P}_{1}=\mathcal{G}_{c, i}^{(1)}\left[N_{\mathbf{q}}^{\mathrm{op},-} \delta\left(X_{1}^{\mathrm{op},-}\right)+N_{\mathbf{q}}^{\mathrm{op},+} \delta\left(X_{1}^{\mathrm{op},+}\right)\right], \\
\mathcal{P}_{1}=\frac{\alpha_{0}^{2}}{8 \alpha_{c}^{2}} \mathcal{G}_{c, i}^{(2)}\left[N_{\mathbf{q}}^{\mathrm{op},-} \delta\left(X_{2}^{\mathrm{op},-}\right)+N_{\mathbf{q}}^{\mathrm{op},+} \delta\left(X_{2}^{\mathrm{op},+}\right)\right],
\end{gathered}
$$

with $N_{\mathbf{q}}^{\mathrm{op}, \pm}=N_{\mathbf{q}}^{\mathrm{op}}+1 / 2 \pm 1 / 2$, in which $N_{\mathbf{q}}^{\mathrm{op}}$ is the Bose distribution function for optical phonon. The arguments of $\delta$ functions are

$$
X_{k}^{\mathrm{op}, \pm}=E_{c, v} \pm \hbar \omega_{\mathrm{op}}-k \hbar \Omega .
$$

Finally, the Lorentz width for optical phonon scattering is $\left(\Gamma_{c, v}^{ \pm, \text {op }}\right)^{2}=\left(\hbar D_{0}^{2} / 4 \pi \rho \omega_{\mathrm{op}} \alpha_{c}^{2}\right) \mathcal{G}_{c, v}^{(0)} N_{\mathbf{q}}^{\mathrm{op}, \pm} \delta_{s, s^{\prime}}$, which is dependent on the LLs.

\section{RESULTS AND DISCUSSION}

\section{A. Two-particle scattering case}

In Fig. 2, the photon energy dependence of the valley and spin breakdown parts and the total MOAC are depicted in silicene and germanene for three cases of electric fields: $\Delta_{z}=0,0.5 \lambda_{\mathrm{SO}}$, and $\lambda_{\mathrm{sO}}$, with $M_{z} \neq 0$. While $\Delta_{z}=0$ stands for the case of without electric field, the latter are chosen to present the two special phases of silicene and germanene: the case of $\Delta_{z}=0.5 \lambda_{\text {SO }}$ refers to the TI phase while the case of $\Delta_{z}=\lambda_{\text {So }}$ is for the VSPM phase [46]. The momentum matrix element in Eq. (14) guarantees that the optical transitions satisfy the selection rule of $n^{\prime}=n \pm 1$, which has been observed in silicene [64] and also in other 2D materials such as graphene [65], topological insulator [66], phosphorene [67], and the TMDCs $[68,69]$. In Fig. 2, the main peaks are due to the transitions between the 0LL and the 1LL. The values 

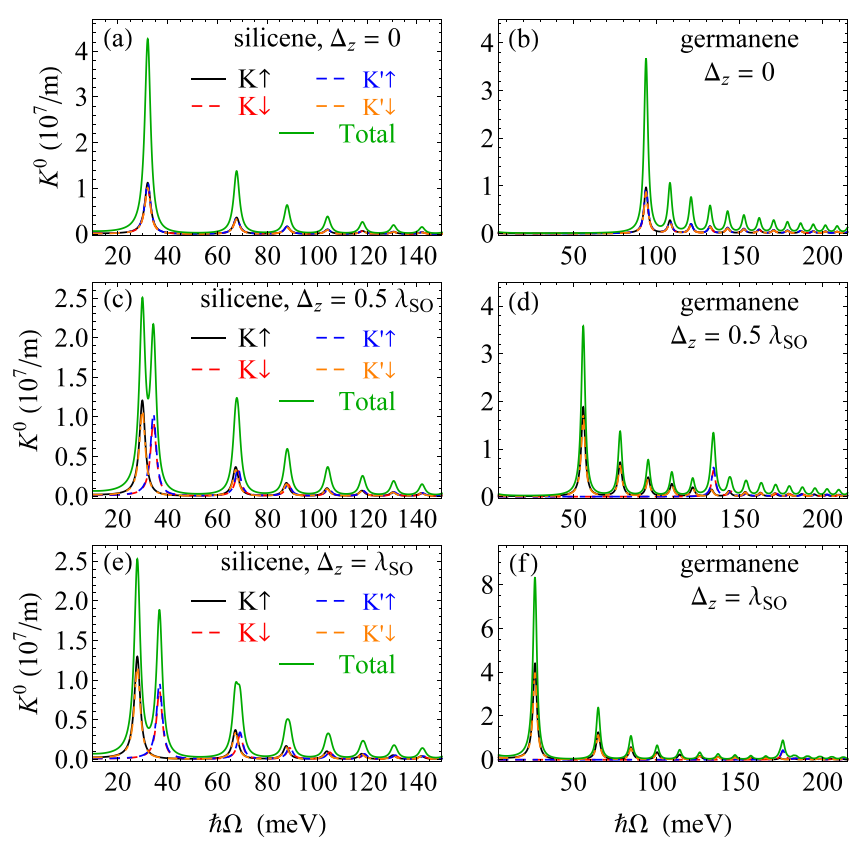

FIG. 2. The valley and spin breakdown parts and the total MOAC as a function of photon energy, in (a), (c), (e) silicene and (b), (d), (f) germanene at the field $B=2 \mathrm{~T}, \gamma=\sqrt{B}(\mathrm{meV})$, and $M_{z} \neq 0$.

of the peak intensities are largest at the transition from the OLL and then reduce when $n$ increases due to the quantity $(\sqrt{n+1}-\sqrt{n})$ as depicted in Eq. (14) of the momentum matrix element. In the absence of electric field [see Figs. 2(a) and 2(b)], although there is a separation between LLs due to spins up and down in each valley $K$ and $K^{\prime}$ as shown in Figs. 1(e) and 1(f), the LL spacings due to spins up and down in the given valley are equal. Consequently, the absorption peaks due to all four valley and spin breakdown transition parts are in the same position, leading to the enhancement of the total MOAC. In the presence of electric field shown in Figs. 2(c) and 2(d) for the TI phase and Figs. 2(e) and 2(f) for the VSPM phase, each absorption peak in the previous case is split into two due to the Zeeman effect. The separation between the two peaks would be larger when the $\Delta_{z}$ increases. This can be explained from the influence of the electric field on the LLs spectrum as follows: With the increase of $\Delta_{z}$ the bandgap in $K \uparrow$ and $K^{\prime} \downarrow$ cases are reduced while those in $K \downarrow$ and $K^{\prime} \uparrow$ are enhanced. Therefore, when the $\Delta_{z}$ increase, the peaks due to $K \uparrow\left(K^{\prime} \downarrow\right)$ are shifted to the lower-energy region (the left-hand side) while the peaks due to $K \downarrow\left(K^{\prime} \uparrow\right)$ are shifted to higher-energy region (also see Fig. 3 for more clarity). Since the separation of the peaks is proportional to the $\Delta_{z}$, these terms in the TI phase are smaller than those in the VSPM phase in both silicene and germanene. This reveals that the spin and valley effects in the TI phase are weaker than that in the VSPM phase. Besides, because of its stronger SOC strength, the separation of the peaks in germanene is larger than in silicene. This also shows that the spin and valley effects in germanene are much stronger in comparison to those in silicene.

In Fig. 3, we show the total MOAC caused by the main transitions (the peaks due to transitions including the 0LL) as a function of $\hbar \Omega$ for three values of $B$ in three cases: $\Delta_{z}=0$,
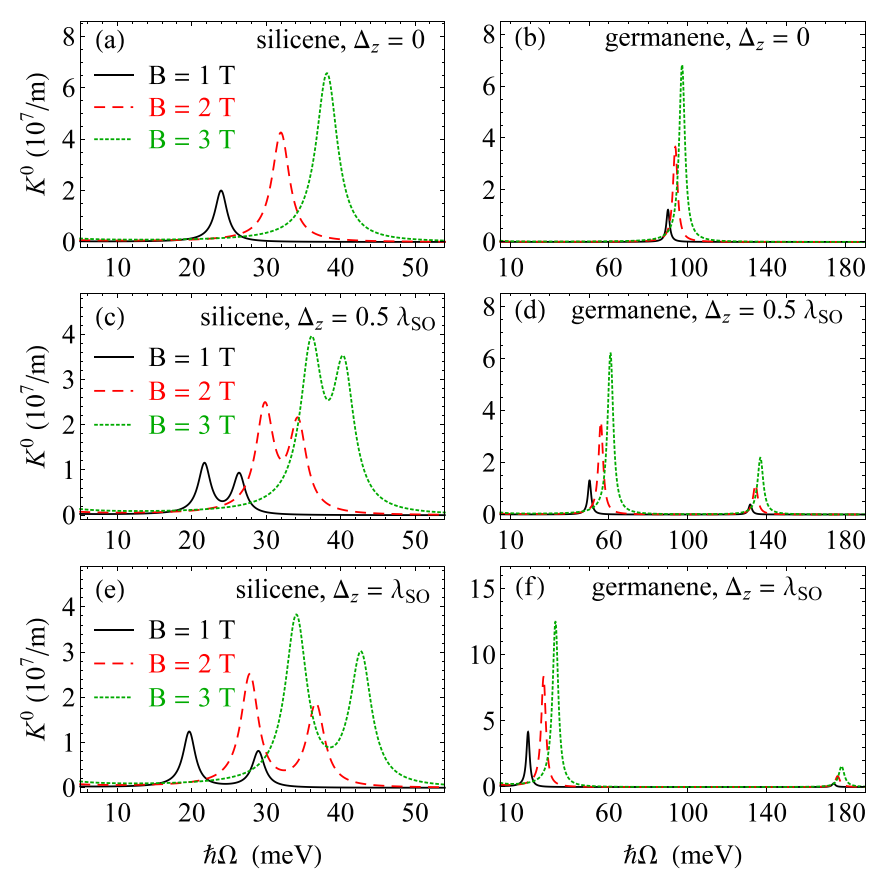

FIG. 3. The photon energy-dependent MOAC due to the main transition for different values of $B$ and $\Delta_{z}$ in (a), (c), (e) silicene and (b), (d), (f) germanene. The results are evaluated for $\gamma=\sqrt{B}$ (meV) and $M_{z} \neq 0$.

$0.5 \lambda_{\mathrm{SO}}$, and $\lambda_{\mathrm{SO}}$. The values of absorbed photon energies are determined from the selection rule

$$
\hbar \Omega=\left|E_{1, s}^{\tau, p}-E_{0, s}^{\tau}\right|
$$

For $\Delta_{z}=\lambda_{\text {So }}$ [Fig. 3(e) and 3(f)], to each value of the field $B=1,2,3 \mathrm{~T}$ the corresponding peak positions for $K \uparrow$ (and also for $K^{\prime} \downarrow$ ), for example, are $\hbar \Omega=19.68,27.83$, and $34.08 \mathrm{meV}(19.02,26.90$, and $32.95 \mathrm{meV})$ in silicene (germanene), respectively. The corresponding peak positions for $K \downarrow$ (and also for $K^{\prime} \uparrow$ ) in silicene (germanene) are $\hbar \Omega=28.97,36.70$, and $42.76 \mathrm{meV}(174.08,176.11$, and $178.10 \mathrm{meV})$. It is clear that when the magnetic field increases, the MOAC displays a blue shift in its position and an enhancement in its intensity. This result is general and has been also observed in other $2 \mathrm{D}$ systems such as graphene [28,60,70,71], silicene [46], and monolayer $\mathrm{MoS}_{2}$ [29]. The blue shift is the consequence of the increase of LLs spacing while the increase of the peak intensity is the result of the reduction of the magnetic length $\alpha_{c}$ with an increase of $B$. One can see from Eq. (13) that the MOAC is proportional to $\alpha_{c}^{-5} \sim B^{5 / 2}$. Therefore, with the increasing field, the MOAC's intensity increases rapidly by the law of $B^{5 / 2}$ as observed in Fig. 3. Besides, the very similar behavior of the absorption spectrum in silicene and germanene is the consequence of their graphenelike LL spectra under the influence of an electric field. It is noted that this result only shows up in the $K \uparrow$ and $K^{\prime} \downarrow$ cases at $\Delta_{z}=\lambda_{\text {so }}$ because the electric field cancels the SOC term only in these cases. The slightly small values of the absorbed photon energies in germanene in comparison to those in silicene is explained by the slightly small value of the Fermi velocity in germanene in comparison to that in silicene. Because, in these cases, we have the product $\tau s=1$, 

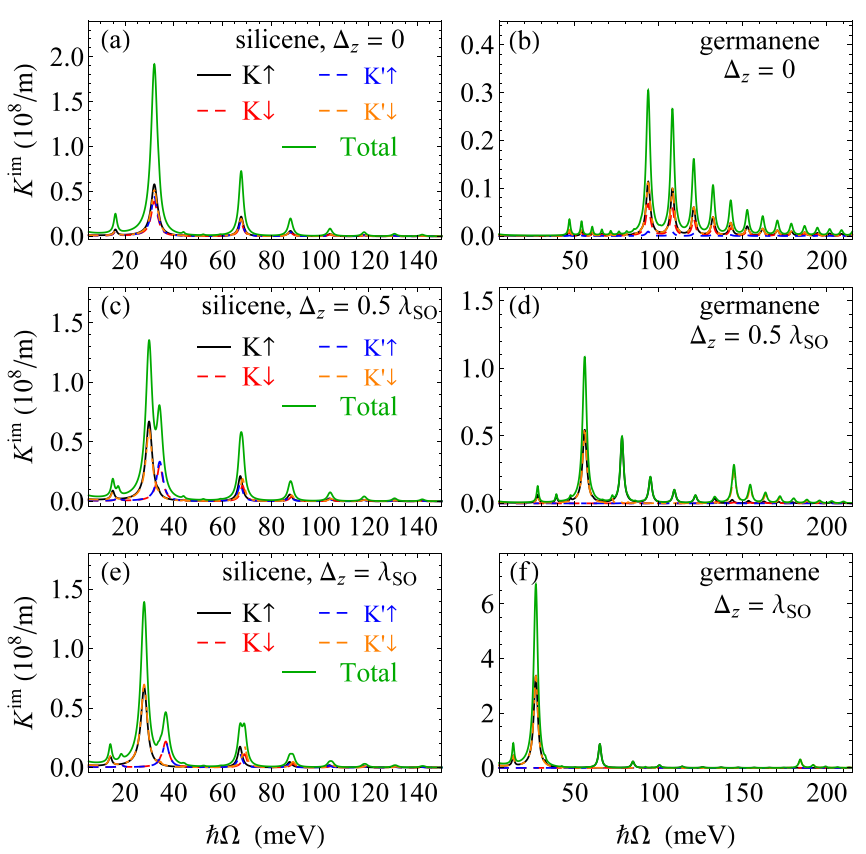

FIG. 4. The MOAC due to impurity-scattering in (a), (c), (e) silicene and (b), (d), (f) germanene as a function of photon energy at $B=2 \mathrm{~T}, T=4 \mathrm{~K}$, and $M_{z} \neq 0$.

therefore, $\Delta_{\tau, s}^{z}=0$, the graphenelike LL spectra of silicene and germanene are reduced to $E_{1, s}^{\tau, p}=p \hbar \omega_{c}+s M_{z}$ and $E_{0, s}^{\tau}=$ $s M_{z}$, which result in $\left|E_{1, s}^{\tau, p}-E_{0, s}^{\tau}\right|=\hbar \omega_{c}=v_{F}(2 e B / \hbar)^{1 / 2}$, being proportional to the Fermi velocity.

\section{B. Three-particle scattering case}

\section{Carrier-photon-impurity scattering}

Figure 4 depicts the MOAC due to impurity scattering as a function of $\hbar \Omega$ in Figs. 4(a), 4(c) silicene and 4(b), 4(d) germanene. The results are evaluated for the valley and spin breakdown parts as well as the total MOAC for three cases of electric field: $\Delta_{z}=0,0.5 \lambda_{\mathrm{so}}$, and $\lambda_{\text {so }}$. Similar to the case of two-particle scattering (see Fig. 2), here we also observe a series of peaks in which the highest (main) peak is generated by the transition between the 0LL and the 1LL. The other peaks come from the transitions between the higher-order LLs, whose peak values gradually reduce with increasing $n$. In the absence of the electric field [see Figs. 4(a) and 4(b)], the peak positions due to four valley and spin breakdown parts coincide but the peak intensities are different. The peak intensities of the $K \downarrow\left(K^{\prime} \uparrow\right)$ are slightly smaller than those of the $K \uparrow\left(K^{\prime} \downarrow\right)$ in both silicene and germanene. Besides, with its larger SOC, the band gap of germanene is bigger than that of silicene [see Figs. 1(e) and 1(f)]. Therefore, the absorbed photon energies in germanene are bigger than those in silicene as shown in Figs. 4(a) and 4(b), where MOACs peaks in germanene appear in the higher regime in comparison to those in silicene. When an electric field is taken into account shown in Figs. 4(c) and 4(d) for the TI phase and in Figs. 4(e) and 4(f) for the VSPM phase, the absorption peaks are separated into two opposite directions for spins up and down caused by the Zeeman effect. While the peaks due to $K \uparrow\left(K^{\prime} \downarrow\right)$ move to
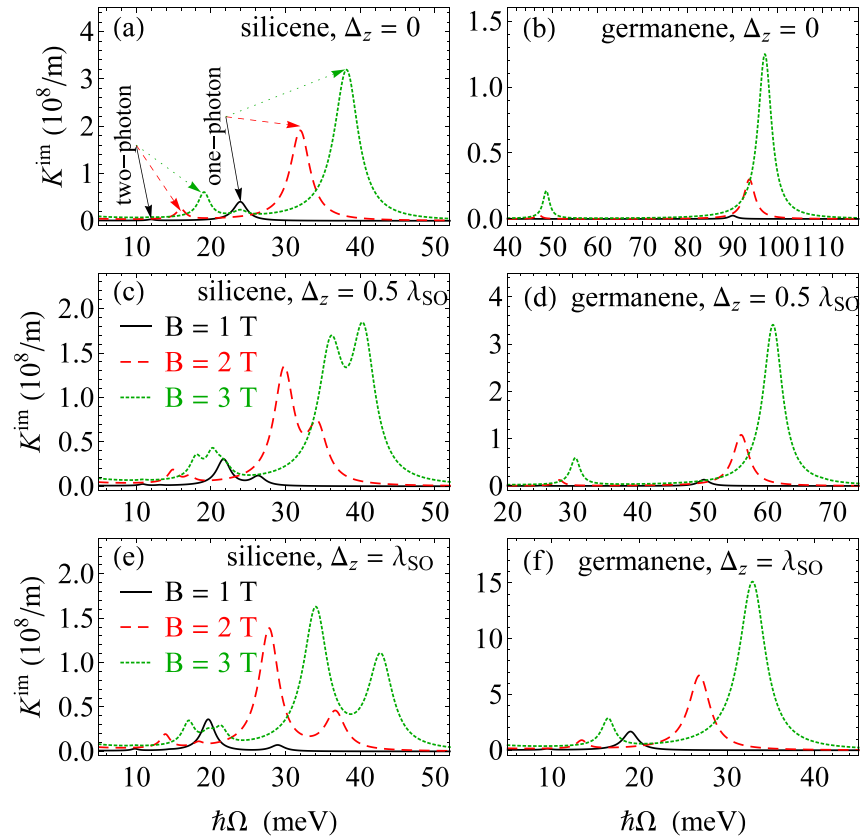

FIG. 5. The photon energy dependence of the total MOAC due to the main transition for different values of magnetic field and $\Delta_{z}$ in (a), (c), (e) silicene and (b), (d), (f) germanene. The results are calculated at $M_{z} \neq 0$ and $T=4 \mathrm{~K}$.

lower energies due to the band gap reducing, the peaks due to $K \downarrow\left(K^{\prime} \uparrow\right)$ move to higher ones in comparison to the case of $\Delta_{z}=0$. Because of its strong SOC effect, the splitting occurs stronger in germanene than that in silicene. A noted feature in Fig. 4 is the appearance of the two-photon absorption peaks whose positions are at the left-hand side of the corresponding one-photon absorption peaks (also see Fig. 5 for more clarity). In comparison to that of the one-photon process, the peak intensity of the two-photon one is smaller but comparable. For example, the ratio of peak intensities between the twoand one-photon processes in Figs. 4(e) and 4(f) is about $13.75 \%(13.55 \%)$ for silicene (germanene), which shows the significant contribution of the two-photon process. We also note that the peak intensities obtained here in silicene and germanene are higher than those in the monolayer TMDCs [31]. The difference in the peak heights of TMDCs and silicene and germanene for the case of impurity scattering is due to the difference in dielectric constants. With smaller dielectric constants, the peak intensities in silicene and germanene are found to be higher than those in monolayer TMDCs.

The effect of varying the magnetic field and electric field are displayed in Fig. 5 for silicene and germanene, respectively. The obtained results here are almost the same as those presented in Fig. 3: with the increase of $B$, the absorption peaks shift their positions to the higher-energy regime and enhance their intensities. The differences between them are the values of the peak intensities and the increasing rate of the peak intensity with the magnetic field. The total MOAC due to impurity scattering is proportional to $\alpha_{c}^{-7} \sim B^{7 / 2}$ instead of $B^{5 / 2}$ shown in the previous case. Besides, similar to the case shown in Fig. 3, here we also found that in the presence of the electric field, the spin degeneracy at a given valley is 

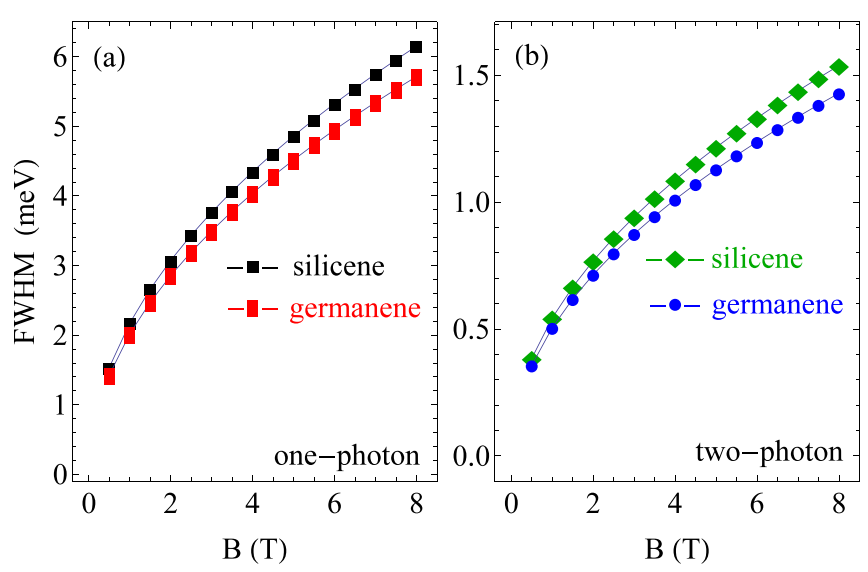

FIG. 6. The magnetic field-dependent FWHM for the (a) oneand (b) two-photon absorption processes. The results are calculated at $\Delta_{z}=\lambda_{\mathrm{SO}}, M_{z} \neq 0$, and $T=4 \mathrm{~K}$.

removed, leading to the splitting of the peaks. The separation of the two peaks increases with the increase of the electric field. Note that the peaks due to the $K \downarrow$ and $K^{\prime} \uparrow$ cases in germanene are in much higher-energy region and are not shown in Figs. 5(b), 5(d), 5(f). Apart from affecting the peak position and the peak intensity, the magnetic field also raises another important feature of the resonant peak, the FWHM, which will be presented in detail in the following.

The magnetic field-dependent FWHM is presented in Fig. 6. Due to its close relationship with the Lorentz width, $\Gamma_{c, v}^{i m}$, which is proportional to $\alpha_{c}^{-1}$ or $B^{1 / 2}$, the FWHM is found to increase with the magnetic field in both silicene and germanene, agreeing with that in conventional low-dimensional systems [72], in graphene [60,70,73], and in $\mathrm{MoS}_{2}$ monolayer [29]. In detail, the magnetic field-dependent FWHM can be calculated as FWHM $(\mathrm{meV})=\beta_{B} \sqrt{B[\mathrm{~T}]}$, in which, the fitted parameters are found in the unit of $\mathrm{meV} / \mathrm{T}^{1 / 2}$ as follows: $\beta_{B}=$ 2.78 (2.02) for the one-photon process and $\beta_{B}=0.54(0.51)$ for the two-photon process in silicene (germanene), respectively. The smallness of the FWHM due to the two-photon process in comparison to that due to the one-photon, which is a universal feature because the contribution of the nonlinear process (the two-photon) is always smaller than the linear one. Moreover, we can easily see from the expression of the Lorentzian width that $\Gamma_{c, v}^{i m} \sim v_{F} / a$. In comparison to that in germanene, this ratio in silicene is bigger. This explains why the FWHM in silicene is bigger than that in germanene as observed in Fig. 6.

\section{Carrier-photon-phonon scattering}

The total MOAC in silicene caused by carrier-phonon scattering versus $\hbar \Omega$ is shown in Fig. 7, where we have taken account of three acoustic and optical modes, i.e., out-of-plane (ZA, ZO), transverse (TA, TO), and longitudinal (LA, LO) modes. The results are evaluated for three values of electric field: $\Delta_{z}=0,0.5 \lambda_{\mathrm{SO}}$, and $\lambda_{\mathrm{SO}}$. Since the electric field affects only the electronic band structure and is independent of the scattering mechanism, the influence of the electric field on the absorption spectrum due to phonon scattering here is similar to those caused by the carrier photon (see Fig. 2) and the
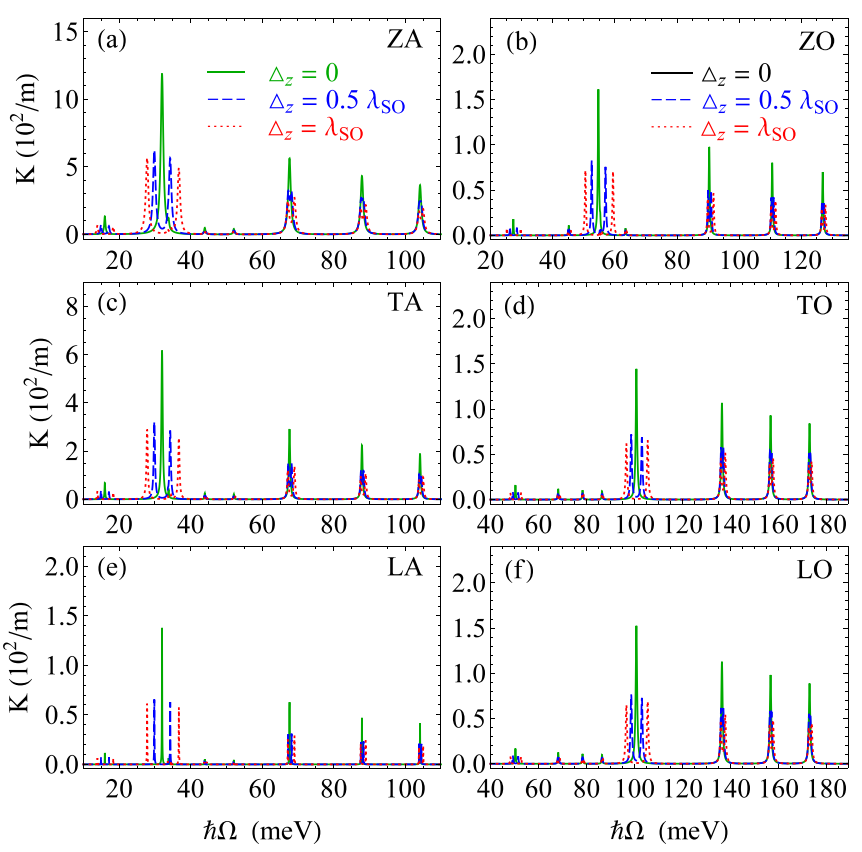

FIG. 7. The photon energy dependence of the total MOAC due to (a), (c), (e) acoustic phonon and (b), (d), (f) optical phonon scatterings in silicene. The results are calculated for three values of the electric field at $B=2 \mathrm{~T}, T=4 \mathrm{~K}$, and $M_{z} \neq 0$.

carrier-photon-impurity (see Fig. 4) scattering mechanisms. Indeed, in the case of $\Delta_{z}=0$, the peaks due to all four spin and valley breakdown parts $\left(K \uparrow, K \downarrow, K^{\prime} \uparrow\right.$, and $\left.K^{\prime} \downarrow\right)$ are located at the same position in both acoustic and optical phonon branches. Meanwhile, in the presence of electric field $\Delta_{z}=0.5 \lambda_{\text {So }}$ and $\Delta_{z}=\lambda_{\text {So }}$, the peaks due to $K \uparrow\left(K^{\prime} \downarrow\right)$ and $K \downarrow\left(K^{\prime} \uparrow\right)$, are separated; they are symmetrical through the peak position of the $\Delta_{z}=0$ case. These displacements of the resonant peaks are equal to $\Delta_{z}$, i.e., the stronger the electric field is the larger the displacements are. In comparison to other scattering mechanisms (see Figs. 2 and 4), the values of MOAC due to phonon scattering are much smaller, being in agreement with the previous prediction in monolayer TMDCs [31]. This is because the illustrations in Ref. [31] and in the present work are for $T=4 \mathrm{~K}$. At this temperature, scattering by phonons is much smaller than that due to impurity for both the systems, which is generally true for a system with disorders.

For the particular case of acoustic phonon interaction, since the MOAC intensity for different phonon modes is mainly proportional to the ratio $D_{1}^{2} / v_{s}^{2}$ [see Eq. (24)], with the highest value of this ratio (see Table II), ZA mode displays the highest MOAC intensity; the next is of TA mode, while the LA

TABLE II. Material parameters for silicene [32,45].

\begin{tabular}{lcccccc}
\hline \hline & ZA & TA & LA & ZO & TO & LO \\
\hline$v_{s}\left(\times 10^{3} \mathrm{~m} / \mathrm{s}\right)$ & 0.63 & 5.4 & 8.8 & - & - & - \\
$D_{1}(\mathrm{eV})$ & 2.0 & 8.7 & 3.2 & - & - & - \\
$D_{0}\left(\times 10^{9} \mathrm{eV} / \mathrm{m}\right)$ & - & - & - & 6.3 & 18.0 & 19.0 \\
$\hbar \omega_{\mathbf{q}, v}(\mathrm{meV})$ & 0 & 0 & 0 & 22.7 & 68.8 & 68.8 \\
\hline \hline
\end{tabular}




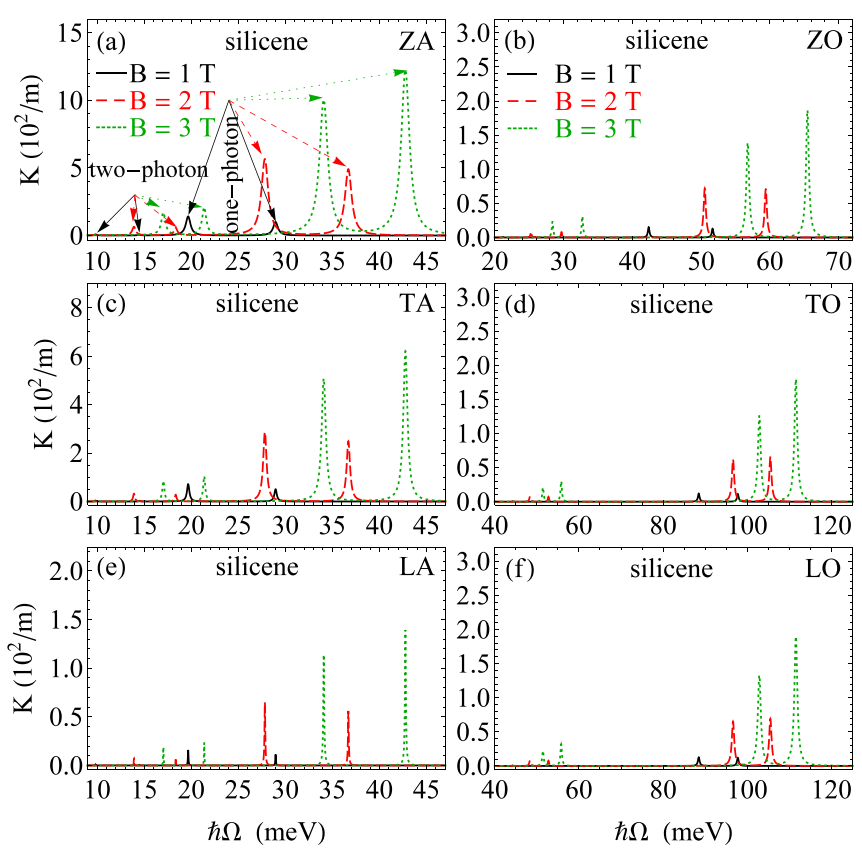

FIG. 8. The photon energy dependence of the total MOAC due to the main transitions in silicene for different values of $B$. The panels (a), (c), (e) and (b), (d), (f) are for acoustic and optical phonon scatterings, respectively. The results are calculated at $\Delta_{z}=\lambda_{\text {so }}$, $M_{z} \neq 0$, and $T=4 \mathrm{~K}$.

mode shows the lowest intensity. This result is in line with that reported for the electron scattering rates in silicene [32]. However, the positions of the MOAC peaks are the same in all three acoustic phonon modes, because the resonant condition, $k \hbar \Omega=E_{c, v}$ is independent of the acoustic phonon modes. Meanwhile, in the case of the optical phonon interaction, the MOAC intensity caused by ZO mode displays the highest. Since the phonon energies of the TO and LO modes are bigger than that of the ZO mode (see Table II), the absorption spectra due to the TO and LO modes shift to higher-energy regime in comparison to that of the $\mathrm{ZO}$ mode.

The magnetic field effect on the MOAC in silicene caused by phonon interaction is shown in Fig. 8. When the magnetic field increases, the MOACs increase in their intensities and shift to the higher-energy region in their positions for all modes of acoustic and optical phonons. This is similar to that in the previous scattering mechanisms as shown in Figs. 3 and 5, and is in agreement with that obtained in graphene $[60,70]$ and in monolayer TMDCs [31], and with the optical conductivity in silicene [46].

In Fig. 9, we show the FWHM in silicene versus magnetic field for different phonon modes. Similar to the previous case (see Fig. 6), a usual $\sqrt{B}$-dependent FWHM has been also found in this case. This reveals that the magnetic fielddependent FWHM is independent of the scattering mechanism. In comparison to the impurity interaction [see Fig. 6(a)] at the low temperature $(T=4 \mathrm{~K})$, the FWHM due to phonon interaction is about from 4 (for ZA) to 37 (for LA) times smaller in value. In silicene, as seen in Fig. 9, the electronphonon interaction contribution to the FWHM increases in the following order: $\mathrm{LA}, \mathrm{ZO}$, TO, LO, TA, and $\mathrm{ZA}$, conforming to a previous work reported for the scattering rate [32] and the
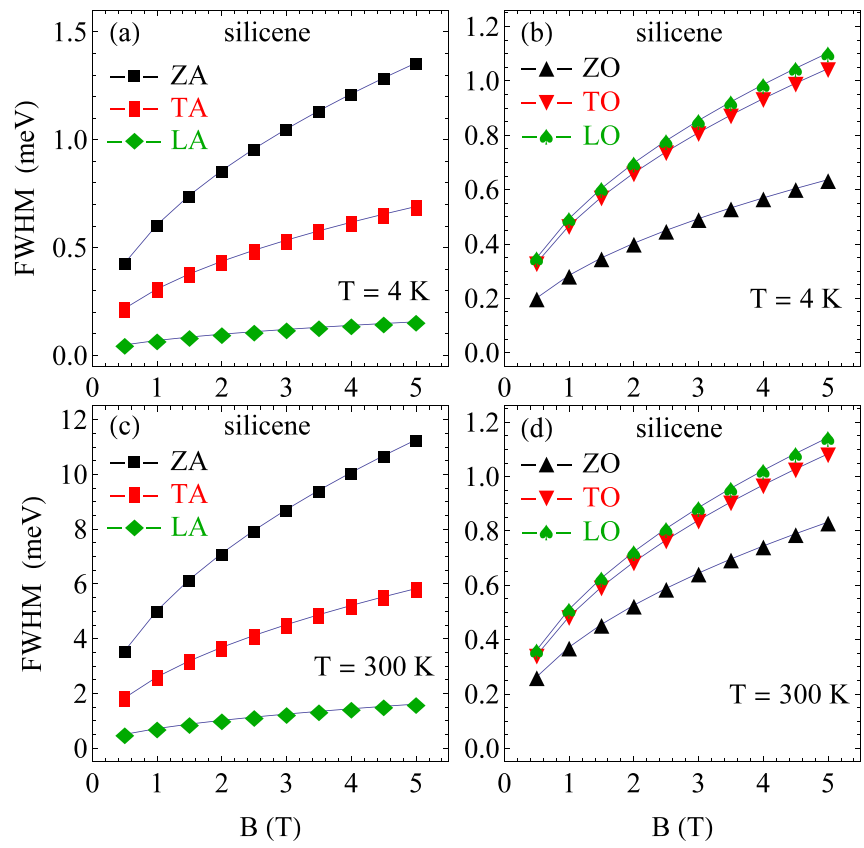

FIG. 9. The magnetic field dependent FWHM of the one-photon peaks due to the acoustic (a), (c) and optical (b), (d) phonon scatterings, respectively. The other parameters are the same as in Fig. 8.

electronic linewidth [74]. The dominant contribution of the ZA branch is caused by its large coupling strength through $D_{1}^{2} / v_{s}^{2}$ [32], which is different from that in graphene [75]. The increased electron scattering by ZA phonons in silicene in comparison to that in graphene can be attributed to its buckled atomic structure. In more detail, it is well known that the FWHM has a close relationship with the Lorentzian width, which is dependent on phonon modes. For the acoustic phonon, the phonon mode dependence of the Lorentzian width is mainly governed by the ratio of $D_{1}^{2} / v_{s}^{2}$. With the highest ratio, the ZA mode displays the biggest FWHM whereas the LA mode expresses the smallest among three acoustic phonon modes. Meanwhile, among the three optical phonon modes, the LO mode displays the highest FWHM caused by its strongest ratio of $D_{0}^{2} / \hbar \omega_{\mathrm{op}}$. Moreover, the increase of the FWHM, in comparison between $T=4 \mathrm{~K}$ [see Figs. 9(a) and 9(b)] and $300 \mathrm{~K}$ [see Figs. 9(c) and 9(d)], is attributed to the enhanced electron-phonon interaction strength when the temperature increases.

The FWHM can be used to estimate the carrier mobility, $\mu_{\lambda}$, using the Landau broadening expression [62]

$$
\Gamma_{\lambda}(B)=\frac{e v_{F}}{\pi} \sqrt{\frac{2 \alpha_{\Gamma} B}{n_{e} \mu_{\lambda}}},
$$

with $\alpha_{\Gamma}=0.1$ being the broadening parameter. Using FWHM data shown in Figs. 9(c) and 9(d) we will obtain the individual contribution of phonon branches [in the unit of $\mathrm{cm}^{2} /(\mathrm{V} \mathrm{s})$ ] as follows: $\mu_{\mathrm{LA}}=1.523 \times 10^{4}, \quad \mu_{\mathrm{TA}}=1.167 \times 10^{3}$, $\mu_{\mathrm{ZA}}=313, \quad \mu_{\mathrm{LO}}=3.031 \times 10^{4}, \quad \mu_{\mathrm{TO}}=3.377 \times 10^{4}$, and $\mu_{\mathrm{ZO}}=5.72 \times 10^{4}$ at $T=300 \mathrm{~K}$ and $n_{e}=3 \times 10^{12} \mathrm{~cm}^{-2}$. These values of $\mu_{\mathrm{LA}}$ and $\mu_{\mathrm{TA}}$ are in agreement with those in previous work [42], but are slightly smaller than those of Shao et al. [76]. Besides, the mobility due to ZA mode is one 
TABLE III. Material parameters for germanene [41,79,80].

\begin{tabular}{lcc}
\hline \hline & acoustic phonon & optical phonon \\
\hline$v_{s}\left(\times 10^{3} \mathrm{~m} / \mathrm{s}\right)$ & 5.31 & - \\
$D_{1}(\mathrm{eV})$ & 4.4 & - \\
$\hbar \omega_{\mathbf{o p}}(\mathrm{meV})$ & - & 37.0 \\
$D_{0}\left(\times 10^{10} \mathrm{eV} / \mathrm{m}\right)$ & - & 1.26 \\
\hline \hline
\end{tabular}

to two orders of magnitude lower than that of the remaining modes. This is due to the strong coupling of the ZA mode in comparison to the others [32,43]. Furthermore, using the Matthiessen's rule $\mu^{-1}=\sum_{\lambda}\left(\mu_{\lambda}\right)^{-1}$ with $\lambda$ being the phonon mode index ( $\lambda=$ ZA, TA, LA, ZO, TO, and LO) we obtain the resultant mobility of silicene of $\mu=1015 \mathrm{~cm}^{2} /(\mathrm{V} \mathrm{s})$ at $T=300 \mathrm{~K}$ and $n_{e}=3 \times 10^{12} \mathrm{~cm}^{-2}$ for the without out-of-plane branches (ZA and ZO) cases. This is almost two orders of magnitude smaller than that of graphene [43] and is in agreement with that of silicene, where the mobility is obtained in the range of $10-1200 \mathrm{~cm}^{2} /(\mathrm{V} \mathrm{s})$ at $T=300 \mathrm{~K}$ for the case of neglecting the ZA and ZO branches [32,77]. If the out-of-plane branches are included, the resultant mobility at the given temperature will be found to reduce down to $238.2 \mathrm{~cm}^{2} /(\mathrm{V} \mathrm{s})$. This result is significantly supported by an experimental observation, where the mobility measured for silicene devices achieves a value of $100 \mathrm{~cm}^{2} /(\mathrm{V} \mathrm{s})$ at $T=300 \mathrm{~K}[78]$.

The above results can also apply to germanene with the input parameters being given in Table III. In Fig. 10, we show the MOAC in germanene versus photon energy for different magnetic fields in both with and without electric field. The mainstream of the increase in the total MOAC intensities and the blue shift in the peak positions with the increase of $B$ is also observed in germanene, similar to those in silicene shown in Fig. 8. The peak positions appeared here satisfy the
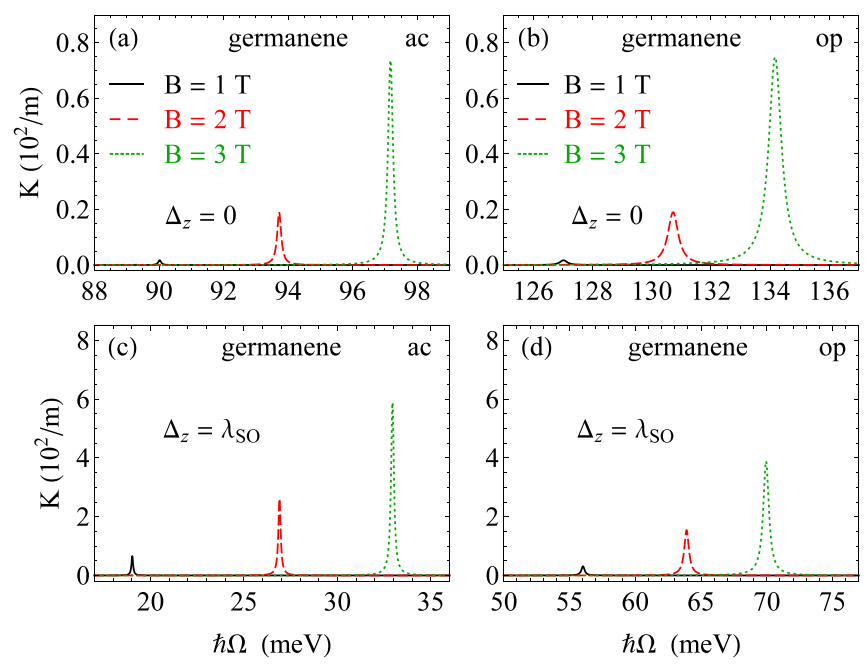

FIG. 10. The photon energy dependence of the total MOAC due to the main transitions in germanene for different magnetic fields. The symbols ac (op) denotes the acoustic (optical) phonon. The results are evaluated at $T=4 \mathrm{~K}$, and only for one-photon absorption process.

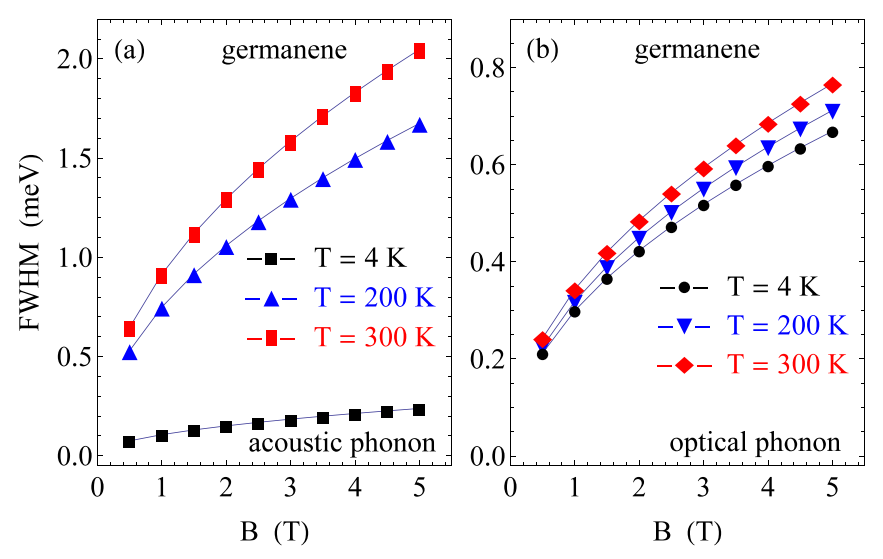

FIG. 11. The magnetic field-dependent FWHM of the onephoton peaks due to the (a) acoustic and (b) optical phonon scatterings, respectively. The results are evaluated for $K \uparrow$ and $\Delta_{z}=\lambda_{\text {so }}$, $M_{z} \neq 0$ case.

condition

$$
k \hbar \Omega=E_{c, v}+\hbar \omega_{\mathrm{ac} / \mathrm{op}},(k=1),
$$

which are derived from the energy conservation law, i.e., the arguments of $\delta$ functions in Eqs. (24) and (25) are equal to zero. For example, at $\Delta_{z}=0$, with each value of the magnetic field of 1,2 , and $3 \mathrm{~T}$, the peak positions due to acoustic (optical) phonon are at 90.02, 93.72, and 97.17 meV (127.02, 130.72, and $134.17 \mathrm{meV}$ ), respectively. Meanwhile, in the case of $\Delta_{z}=\lambda_{\text {SO }}$, since the SOC terms of the $K \uparrow$ and $K^{\prime} \downarrow$ have been canceled by the electric field, the LLs spectrum of germanene reduced to that of the pristine graphene [see Fig. 1(h)]. Consequently, the peak positions shift to the lowerenergy regime in comparison to those of the case of $\Delta_{z}=0$. The corresponding peak positions for the acoustic (optical) phonon interaction are 19.02, 26.90, and $32.95 \mathrm{meV}$ (56.02, 63.90, and $69.95 \mathrm{meV}$ ), respectively. We can also see that in both cases with and without electric field, the peak positions due to the optical phonon interaction are always at the righthand side of those due to the acoustic phonon with the shift being exactly equal to the optical phonon energy.

The FWHM due to phonon interactions in germanene as a function of $B$ for different temperatures is shown in Fig. 11. Similar to silicene shown in Fig. 9, the $\sqrt{B}$-dependent FWHM is also observed for the phonon interaction in germanene. The fitted expression for the FWHM can be expressed as FWHM $(\mathrm{meV})=\alpha_{B} \sqrt{B[\mathrm{~T}]}$, where the fitted parameters at $T=4,200$, and $300 \mathrm{~K}$ are $\alpha_{B}=0.11,0.75$, and $0.92 \mathrm{meV} / \mathrm{T}^{1 / 2}$ for the acoustic phonon interaction and $\alpha_{B}=0.30,0.32$, and $0.34 \mathrm{meV} / \mathrm{T}^{1 / 2}$ for the optical phonon interaction, respectively. It is clear that the FWHM increases with temperature with different rates depending on the phonon branch. This rate is bigger for the acoustic phonon in comparison to the optical phonon interaction, being in agreement with what happened in the monolayer transition-metal dichalcogenides [31]. Using these FWHM data and the equation (30), the mobility of germanene can be evaluated in the unit of $\mathrm{cm}^{2} /(\mathrm{V} \mathrm{s})$ as $\mu_{\mathrm{ac}}=8.846 \times 10^{3}$ and $\mu_{\mathrm{op}}=6.297 \times 10^{4}$ at $T=300 \mathrm{~K}$ and $n_{e}=3 \times 10^{12} \mathrm{~cm}^{-2}$, which leads to a resultant mobility value of $\mu=7.756 \times 10^{3} \mathrm{~cm}^{2} /(\mathrm{V} \mathrm{s})$. In 
comparison, the mobilities due to the acoustic phonon interaction in germanene have achieved at $\mu_{\mathrm{TA}}=8.82 \times 10^{4}$ $\mathrm{cm}^{2} /(\mathrm{V} \mathrm{s})$ and $\mu_{\mathrm{LA}}=1.34 \times 10^{5} \mathrm{~cm}^{2} /(\mathrm{V} \mathrm{s})[42]$, which leads to a resultant mobility of $\mu_{\mathrm{ac}}=5.319 \times 10^{4} \mathrm{~cm}^{2} /(\mathrm{V} \mathrm{s})$. In another work, Ye et al. [37] showed that the electron mobility due to acoustic phonon interaction in germanene can reach a value of $6.24 \times 10^{5} \mathrm{~cm}^{2} /(\mathrm{V} \mathrm{s})$, which is still very high in comparison to our result. Besides, the recent studies reported that the mobility of germanene can achieve up to $2.2 \times 10^{5}$ $\mathrm{cm}^{2} /(\mathrm{V} \mathrm{s})$ or in the range of $1-9 \times 10^{5} \mathrm{~cm}^{2} /(\mathrm{V} \mathrm{s})$ if the substrate $M X(M=\mathrm{Ga}$, In; $X=\mathrm{S}, \mathrm{Se}, \mathrm{Te})[81]$ or the $\mathrm{CdI}_{2}$-type materials [82] are included, respectively.

\section{SUMMARY AND CONCLUSIONS}

We have studied the magneto-optical absorption properties of the 2D honeycomb group-IV crystals silicene and germanene in the presence of magnetic and electric fields including the spin and valley effects. The results are evaluated for different types of electron interactions: (i) electron-photon only; (ii) electron-photon-impurity; and (iii) electron-photonphonon. The electric field and Zeeman term have a strong effect on the LLs of silicene and germanene: from quadruple spin and valley degenerate in the case of $\Delta_{z}=M_{z}=0$ to nondegenerate in the case of $\Delta_{z} \neq 0, M_{z} \neq 0$ for all LLs with $n \neq 0$. Therefore, these two quantities affect strongly the absorption spectrum in these two materials.

A series of peaks has been observed in the absorption spectra in all scattering mechanisms in which the main peak is generated by the transition between the 0LL and 1LL and displays the highest intensity. The high-order peaks come from the transitions between the higher-order LLs, whose peak values gradually reduce with the increase of LL index $n$. In the absence of electric field, the peak positions due to $K \uparrow$ $\left(K^{\prime} \downarrow\right)$ and $K \downarrow\left(K^{\prime} \uparrow\right)$ in each material are coincident but the peaks in germanene appear in the higher regime in comparison to those in silicene due to its stronger SOC strength. When an electric field is taken into account, the absorption peaks are separated into two opposite directions for spins up and down under the Zeeman effect. Because of its strong SOC effect, the splitting is stronger in germanene than that in silicene

Among the scattering mechanisms, the peak value due to impurity scattering is the highest (for the present choice impurity density), followed by that of the two-particle scattering, while the phonon scattering shows the lowest in both materials. For the effect of different phonon modes, the ZA mode in silicene dominates the others due to its largest coupling strength. This is attributed to its buckled atomic structure, which could not exist in graphene. When the ZA mode is included, the resultant mobility of silicene is estimated down from $1218 \mathrm{~cm}^{2} /(\mathrm{V} \mathrm{s})$ to $285.8 \mathrm{~cm}^{2} /(\mathrm{V} \mathrm{s})$ at $T=300 \mathrm{~K}$, which is significantly supported by the experimental observation.

\section{ACKNOWLEDGMENTS}

This research is funded by the Vietnam National Foundation for Science and Technology Development (NAFOSTED) under Grant No. 103.01-2019.11 and the Belarusian National Research Program "Convergence-2020".
[1] K. S. Novoselov, A. K. Geim, S. V. Morozov, D. Jiang, Y. Zhang, S. V. Dubonos, I. V. Grigorieva, and A. A. Firsov, Science 306, 666 (2004).

[2] S. Cahangirov, M. Topsakal, E. Aktürk, H. Sahin, and S. Ciraci, Phys. Rev. Lett. 102, 236804 (2009).

[3] H. Sahin, S. Cahangirov, M. Topsakal, E. Bekaroglu, E. Akturk, R. T. Senger, and S. Ciraci, Phys. Rev. B 80, 155453 (2009).

[4] P. Vogt, P. De Padova, C. Quaresima, J. Avila, E. Frantzeskakis, M. C. Asensio, A. Resta, B. Ealet, and G. Le Lay, Phys. Rev. Lett. 108, 155501 (2012).

[5] L. Meng, Y. Wang, L. Zhang, S. Du, R. Wu, L. Li, Y. Zhang, G. Li, H. Zhou, W. A. Hofer, and H.-J. Gao, Nano Lett. 13, 685 (2013)

[6] L. C. Lew Yan Voon and G. G. Guzmán-Verri, MRS Bull. 39, 366 (2014).

[7] C.-C. Liu, W. Feng, and Y. Yao, Phys. Rev. Lett. 107, 076802 (2011).

[8] X.-T. An, Y.-Y. Zhang, J.-J. Liu, and S.-S. Li, Appl. Phys. Lett. 102, 043113 (2013).

[9] M. Ezawa, Phys. Rev. B 87, 155415 (2013).

[10] M. Ezawa, New J. Phys. 14, 033003 (2012).

[11] M. Ezawa, Phys. Rev. Lett. 109, 055502 (2012).

[12] M. Ezawa, Phys. Rev. Lett. 110, 026603 (2013).

[13] M. C. Hersam, N. P. Guisinger, and J. W. Lyding, Nanotechnology 11, 70 (2000).
[14] A. Pulkin and O. V. Yazyev, Phys. Rev. B 93, 041419(R) (2016).

[15] P. M. Krstajić, P. Vasilopoulos, and M. Tahir, Phys. Rev. B 94, 085413 (2016).

[16] M. Tahir, P. M. Krstajić, and P. Vasilopoulos, Phys. Rev. B 95, 235402 (2017).

[17] R.-Y. Yuan, Q.-J. Yang, and Y. Guo, J. Phys.: Condens. Matter 30, 355301 (2018).

[18] Q.-J. Yang, R.-Y. Yuan, and Y. Guo, J. Phys. D: Appl. Phys. 52, 335301 (2019).

[19] T. Yokoyama, Phys. Rev. B 87, 241409(R) (2013).

[20] T. Hua, X. Zhai, Z. Yang, S. Wang, and B. Li, Solid State Commun. 244, 43 (2016).

[21] V. Vargiamidis and P. Vasilopoulos, Appl. Phys. Lett. 105, 223105 (2014).

[22] V. Vargiamidis and P. Vasilopoulos, J. Appl. Phys. 117, 094305 (2015).

[23] C. J. Tabert and E. J. Nicol, Phys. Rev. B 87, 235426 (2013).

[24] B. Stébé, E. Feddi, A. Ainane, and F. Dujardin, Phys. Rev. B 58, 9926 (1998).

[25] L. Brey, N. F. Johnson, and B. I. Halperin, Phys. Rev. B 40, 10647(R) (1989).

[26] F. M. Peeters, Phys. Rev. B 42, 1486(R) (1990).

[27] H. V. Phuc, N. D. Hien, L. Dinh, and T. C. Phong, Superlattices Microstruct. 94, 51 (2016). 
[28] B. D. Hoi, L. T. T. Phuong, and T. C. Phong, J. Appl. Phys. 123, 094303 (2018).

[29] C. V. Nguyen, N. N. Hieu, N. A. Poklonski, V. V. Ilyasov, L. Dinh, T. C. Phong, L. V. Tung, and H. V. Phuc, Phys. Rev. B 96, 125411 (2017).

[30] G. Catarina, J. Have, J. Fernández-Rossier, and N. M. R. Peres, Phys. Rev. B 99, 125405 (2019).

[31] N. D. Hien, C. V. Nguyen, N. N. Hieu, S. S. Kubakaddi, C. A. Duque, M. E. Mora-Ramos, L. Dinh, T. N. Bich, and H. V. Phuc, Phys. Rev. B 101, 045424 (2020).

[32] X. Li, J. T. Mullen, Z. Jin, K. M. Borysenko, M. Buongiorno Nardelli, and K. W. Kim, Phys. Rev. B 87, 115418 (2013).

[33] P. Borowik, J.-L. Thobel, and L. Adamowicz, Semicond. Sci. Technol. 31, 115004 (2016).

[34] B. I. Abidin, K. H. Yeoh, D. S. Ong, and T. K. Yong, J. Phys. D: Appl. Phys. 50, 425103 (2017).

[35] G. Gaddemane, W. G. Vandenberghe, M. L. Van de Put, E. Chen, and M. V. Fischetti, J. Appl. Phys. 124, 044306 (2018).

[36] R. Rengel, J. M. Iglesias, E. M. Hamham, and M. J. Martín, Semicond. Sci. Technol. 33, 065011 (2018).

[37] X.-S. Ye, Z.-G. Shao, H. Zhao, L. Yang, and C.-L. Wang, RSC Adv. 4, 21216 (2014).

[38] V. A. Margulis, J. Exp. Theor. Phys. 99, 633 (2004).

[39] K. Shakouri, P. Vasilopoulos, V. Vargiamidis, and F. M. Peeters, Phys. Rev. B 90, 125444 (2014).

[40] B. Peng, H. Zhang, H. Shao, Y. Xu, G. Ni, R. Zhang, and H. Zhu, Phys. Rev. B 94, 245420 (2016).

[41] N. J. Roome and J. D. Carey, ACS Appl. Mater. Interfaces 6, 7743 (2014).

[42] M. V. Fischetti and W. G. Vandenberghe, Phys. Rev. B 93, 155413 (2016).

[43] T. Gunst, T. Markussen, K. Stokbro, and M. Brandbyge, Phys. Rev. B 93, 035414 (2016).

[44] T. Stauber, N. M. R. Peres, and F. Guinea, Phys. Rev. B 76, 205423 (2007).

[45] K. H. Yeoh, D. S. Ong, C. H. R. Ooi, T. K. Yong, and S. K. Lim, Semicond. Sci. Technol. 31, 065012 (2016).

[46] C. J. Tabert and E. J. Nicol, Phys. Rev. B 88, 085434 (2013).

[47] M. Ezawa, Phys. Rev. B 86, 161407(R) (2012).

[48] C.-C. Liu, H. Jiang, and Y. Yao, Phys. Rev. B 84, 195430 (2011).

[49] G. Dresselhaus, A. F. Kip, and C. Kittel, Phys. Rev. 98, 368 (1955).

[50] J. Menéndez, M. Noël, J. C. Zwinkels, and D. J. Lockwood, Phys. Rev. B 96, 121201(R) (2017).

[51] P.-H. Shih, Y.-H. Chiu, J.-Y. Wu, F.-L. Shyu, and M.-F. Lin, Sci. Rep. 7, 40600 (2017)

[52] C. M. Wang and X. L. Lei, Phys. Rev. B 92, 125303 (2015).

[53] L. Matthes, P. Gori, O. Pulci, and F. Bechstedt, Phys. Rev. B 87, 035438 (2013).
[54] H. J. G. Meyer, Phys. Rev. 112, 298 (1958).

[55] E. R. Generazio and H. N. Spector, Phys. Rev. B 20, 5162 (1979).

[56] C. T. Giner and M. Antón, Phys. Status Solidi B 133, 563 (1986).

[57] W. Xu, R. A. Lewis, P. M. Koenraad, and C. J. G. M. Langerak, J. Phys.: Condens. Matter 16, 89 (2004).

[58] W. Xu, Phys. Rev. B 57, 12939 (1998).

[59] H. N. Spector, Phys. Rev. B 28, 971 (1983).

[60] H. V. Phuc and N. N. Hieu, Opt. Commun. 344, 12 (2015).

[61] M. P. Chaubey and C. M. Van Vliet, Phys. Rev. B 33, 5617 (1986).

[62] C. M. Wang and X. L. Lei, Phys. Rev. B 87, 235403 (2013).

[63] Y. D. Kuang, L. Lindsay, S. Q. Shi, and G. P. Zheng, Nanoscale 8, 3760 (2016).

[64] C. J. Tabert and E. J. Nicol, Phys. Rev. Lett. 110, 197402 (2013).

[65] V. P. Gusynin, S. G. Sharapov, and J. P. Carbotte, Phys. Rev. Lett. 98, 157402 (2007).

[66] Z. Li and J. P. Carbotte, Phys. Rev. B 88, 045414 (2013).

[67] M. Tahir, P. Vasilopoulos, and F. M. Peeters, Phys. Rev. B 92, 045420 (2015).

[68] M. Tahir, P. Vasilopoulos, and F. M. Peeters, Phys. Rev. B 93, 035406 (2016).

[69] M. Tahir and P. Vasilopoulos, Phys. Rev. B 94, 045415 (2016).

[70] H. V. Phuc and L. Dinh, Mater. Chem. Phys. 163, 116 (2015).

[71] B. Scharf, V. Perebeinos, J. Fabian, and I. Žutić, Phys. Rev. B 88, 125429 (2013).

[72] K. D. Pham, L. V. Tung, D. V. Thuan, C. V. Nguyen, N. N. Hieu, and H. V. Phuc, J. Appl. Phys. 126, 124301 (2019).

[73] Z. Jiang, E. A. Henriksen, L. C. Tung, Y.-J. Wang, M. E. Schwartz, M. Y. Han, P. Kim, and H. L. Stormer, Phys. Rev. Lett. 98, 197403 (2007).

[74] V. N. Popov and P. Lambin, 2D Materials 3, 025014 (2016).

[75] K. M. Borysenko, J. T. Mullen, E. A. Barry, S. Paul, Y. G. Semenov, J. M. Zavada, M. B. Nardelli, and K. W. Kim, Phys. Rev. B 81, 121412(R) (2010).

[76] Z.-G. Shao, X.-S. Ye, L. Yang, and C.-L. Wang, J. Appl. Phys. 114, 093712 (2013).

[77] R. Wang, X. Pi, Z. Ni, Y. Liu, S. Lin, M. Xu, and D. Yang, Sci. Rep. 3, 3507 (2013).

[78] L. Tao, E. Cinquanta, D. Chiappe, C. Grazianetti, M. Fanciulli, M. Dubey, A. Molle, and D. Akinwande, Nat. Nanotechnol. 10, 227 (2015).

[79] P. Chaudhary, A. Salimath, and B. Ghosh, J. Comput. Theor. Nanosci. 11, 2437 (2014).

[80] F. Szmulowicz, Phys. Rev. B 28, 5943 (1983).

[81] Z. Ni, E. Minamitani, Y. Ando, and S. Watanabe, Phys. Chem. Chem. Phys. 17, 19039 (2015).

[82] Z. Ni, E. Minamitani, Y. Ando, and S. Watanabe, Phys. Rev. B 96, 075427 (2017). 